\title{
PRECURSORES CONDUCTUALES INFANTILES DE LOS TRASTORNOS DEL ESPECTRO ESQUIZOFRÉNICO: ESQUIZOFRENIA Y TRASTORNO ESQUIZOTÍPICO DE LA PERSONALIDAD
}

\author{
SERGI BALLESPI, NEUS BARRANTES-VIDAL Y JORDI E. OBIOLS \\ Universidad Autónoma de Barcelona
}

(Recibido el 17 de junio de 1999)

\begin{abstract}
Dada la extensa y creciente aparición de trabajos sobre marcadores bioconductuales de los trastornos del espectro esquizofrénico, hemos pretendido realizar una tarea de síntesis de los datos aportados y orientarla a la cuestión de posibles diferencias en cuanto a precursores de la evolución hacia el trastorno esquizotípico y la esquizofrenia. Después de situar conceptualmente a los estudios de riesgo elevado, se revisan en primer lugar los trabajos clásicos y más recientes sobre marcadores psicobiológicos dentro del paradigma del riesgo genético para la esquizofrenia y, en segundo lugar, se presentan los hallazgos de los trabajos que han tomado como marcadores de riesgo variables no sólo genéticas y que se han centrado en el estudio de las condiciones leves del espectro (esto es, esquizotipia y trastorno esquizotípico de la personalidad). Por último se discuten las limitaciones de los trabajos existentes y también sus implicaciones teóricas y aplicadas.
\end{abstract}

Palabras clave: esquizofrenia, esquizotipla, marcadores conductuales, riesgo genético, riesgo bioconductual.

Behavioural childhood precursors of schizophrenia spectrum disorders: schizophrenia and schizotypal personality disorder

Given the wide and growing appearance of studies on blobehavioural markers of schizophrenia spectrum disorders, we have pursued to synthesise the data focusing on the possible differences in such markers as precursors of development towards schizotypal disorder and schizophrenia. Following a conceptual seting of high-risk studies we first outline classic and recent works about psychobiological markers from the genetic risk paradigm for schizophrenia and, secondly, we present findings coming from studies that have used other than genetic markers and have focused on the analysis of the soft conditions of the spectrum (i.e., schizotypy and schizotypal personality disorder). Lastly, limitations and theoretical and applied implications of these studies are discussed.

Key words: schizophrenia, schizotypy, behavioural markers, genetic risk, biobehavioural risk.

\section{INTRODUCCION}

Podemos interpretar los rasgos esquizotípicos y esquizoides como indicadores de predisposición a la psicosis si nos basamos en un principio en el que ya Bleuler reparó: que la patología grave es precedi-

Correspondencia: Neus Barrantes-Vidal. Departamento de Psicología de la Salud y Psicologia Social, Facultad de Psicología, Edificio B, Universidad Autónoma de Barcelona, 08193 Bellaterra. neus.barrantes@uab.es da a menudo por sintomatología más leve del mismo tipo (Bleuler, 1911/1950). Así, estilos inusuales de personalidad, anomalías del neurodesarrollo y problemas del lenguaje o la motricidad pueden aparecer como precursores tempranos de un cuadro esquizofrénico ulterior (Russell, 1992).

No obstante, hay que sortear la dificultad de distinguir, según la edad, cuándo estos signos pueden considerarse prodrómicos. Esto implica tener en cuenta 
tanto el efecto del desarrollo sobre la psicopatología como el impacto de ésta sobre el desarrollo (Rosenbaum, 1988). Sin ir más lejos, las alucinaciones visuales y táctiles, ocasionalmente observables en preescolares - particularmente en situaciones de ansiedad y estrés(Rothstein, 1981) tienen un pronóstico relativamente benigno, sobre todo cuando son fenómenos hipnagógicos. Igualmente, es difícil considerar como trastornos del pensamiento las frecuentes pérdidas de asociaciones y el pensamiento ilógico que se reflejan en el lenguaje anterior a los 6 años, dada la inhabilidad de la población preescolar para utilizar las nociones de realidad y la lógica adultas (Caplan, 1994).

A partir de estas edades, y hasta la adolescencia, la frecuencia de alucinaciones y delirios que no cumplen criterios diagnósticos de psicosis oscila entre el $2 \%$ y el $30 \%$ según los estudios (Apter, Bleich y Tyano, 1988; Famularo, Kinscheff y Fenton, 1992; Livingston, Lawson y Jones, 1993). La existencia de estos síntomas subclínicos en la infancia puede apoyar lo que Meehl $(1962,1989,1990)$ propone en la Teoría del Déficit Neurointegrativo: que tras la etiología de la esquizofrenia se esconden factores constitucionales cuya expresión puede observarse ya en la infancia.

Rado había acuñado en 1953 el término "esquizotipo" para designar el rango de fenotipos que presuntamente se expresaban a partir del "genotipo esquizofrénico». Posteriormente Meehl propuso que la sintomatología esquizofrénica resulta de la interacción entre las variables de herencia y las de ambiente, entendiendo que lo que se hereda es una predisposición a la fragilidad del sistema nervioso central denominada por él "esquizotaxia", basada en una tendencia de las neuronas a la "hypokrisia» (dispararse o no hacerlo en momentos inapropiados). El resultado de la herencia del «esquizogen» sería la presentación de los síntomas fundamentales de tipo esquizofrénico recogidos en la condición que él denomina "esquizotipia», los cuales pueden llegar a expresarse en forma de cuadro esquizofrénico según la exposición a ciertas variables ambientales. Según este modelo de vulnerabilidad, la predisposición a los trastornos del espectro esquizofrénico se distribuye dicotómicamente en la población normal, y puede ser detectada premórbidamente mediante indicadores conductuales análogos a los criterios diagnósticos del trastorno esquizotípico de la personalidad. Habría, sin embargo, algunos sujetos que presentarían características esquizotípicas no atribuibles a la herencia genética; estos serían "fenocopias», o sea imitaciones del fenotipo esquizotípico que deben ser discriminados del esquizotípico "real» mediante la identificación estadística del «taxon» esquizoide. Por ende, el estudio de los rasgos esquizotípicos de la población normal podría dilucidar algunos aspectos de la predisposición a la esquizofrenia.

Qué mejor, para este propósito, que los estudios de cohortes de alto riesgo, cuya longitudinalidad permite el análisis de las discontinuidades de la psicopatología a lo largo de la vida (Gooding y Iacono, 1995). Este tipo de estudios, de diseño prospectivo, tienen la ventaja de estar libres de ciertos sesgos como que familiares o investigadores sepan de la condición diagnóstica del sujeto, o los asociados a la recogida de información de forma retrospectiva. Además, permiten la sistematicidad y uniformidad de las evaluaciones, con las ventajas que esto supone para conjeturar sobre posibles etiologías, asegurar la detección de todos los casos que presenten patología y corroborar el diagnóstico diferencial en presencia de ambigüedades (Mednick, Parnas y Schulsinger, 1987; Parnas et al., 1993; Erlenmeyer et al.,1995). 
En los estudios de riesgo genético, la condición de riesgo elevado viene determinada por la relación genética con un sujeto afectado. Esto implica que los sujetos con al menos un familiar de primer grado afectado (normalmente uno de los padres) tienen un riesgo de sufrir la patología, superior al de la población normal: unas 10 veces superior en el caso de la esquizofrenia y hasta 40 si son ambos padres los afectados.

No obstante, ante las dificultades de detectar marcadores de vulnerabilidad dada la baja prevalencia de la esquizofrenia (1\%), y teniendo en cuenta que la mayoría de los padres, hermanos o descendencia de esquizofrénicos no presentan la enfermedad, tiene especial importancia la alternativa de distinguir a los sujetos de riesgo mediante variables bioconductuales como son las alteraciones de los movimientos oculares, los déficit atencionales o las desviaciones que la respuesta electrodermal sufre ante algunas tareas experimentales. Así, de forma paralela a la investigación de alto riesgo genético se ha desarrollado en las últimas décadas la estrategia de alto riesgo psicométrico y/o bioconductual, lo que permite estudiar los marcadores candidatos sin la interferencia de factores como la medicación, presencia de sintomatología activa, institucionalización, cronicidad, deterioro $\mathrm{y}$, especialmente, la no necesidad de restringir las muestras a descendientes biológicos.

Podemos clasificar estos marcadores bioconductuales en tres dominios según correspondan a 1) capacidades atencionales o de procesamiento de la información, 2) funcionamiento neuromotor o 3) procesos psicológicos, y debemos distinguirlos de variables etiológicas precoces (complicaciones obstétricas y perinatales) o más tardias (funcionamiento familiar deficiente, separación de los padres, institucionalización), consideradas más acertadamente como factores de riesgo ambiental (Olin y Mednick, 1996).
En el presente trabajo, amparado por la perspectiva del desarrollo, tenemos por objetivo revisar estos precursores bioconductuales, cuyo valor predictivo de los trastornos del espectro esquizofrénico se demuestra en muchos de los casos, y cuyo interés clínico es, en consecuencia, elevado. Con esta finalidad, nos centraremos especialmente en los estudios de alto riesgo más significativos que se han llevado a cabo en las últimas décadas. Todos ellos se centran en las edades que comprenden la niñez y la adolescencia, y en conjunto, están orientados a arrojar algo de luz a cuestiones como estas que siguen: ¿Los individuos que desarrollan un trastorno esquizofrénico en la adultez, son distinguibles de los sujetos controles en su infancia? ¿Cuál es el momento más temprano del desarrollo en que los signos de vulnerabilidad son observables? ¿Cuál es la naturaleza de esas manifestaciones de vulnerabilidad?

\section{PRECURSORES CONDUCTUALES INFANTILES DE LA ESQUIZOFRENIA}

En este apartado se describirán los principales resultados hallados por los estudios pioneros del campo que nos ocupa. Los aspectos metodológicos de todos ellos pueden verse en la tabla 1.

Si bien los métodos retrospectivos permiten distinguir a los sujetos pre-esquizofrénicos cuando más temprano a los 15 años, Walker y Lewine (1990) consiguieron demostrar que los adultos esquizofrénicos exhiben características comportamentales idiosincráticas antes de los 8 años de edad. En su estudio, 19 clínicos experimentados discriminaron, entre sus hermanos, a los cinco niños que a los 20 años cumplieron criterios DSM-III-R para el diagnóstico de la esquizofrenia. Para ello se basaron en grabaciones caseras de la infancia de estos sujetos, películas que constituyen muestras de la conducta 
Tabla 1. Características metodológicas de los principales estudios prospectivos de alto riesgo de esquizofrenia

\begin{tabular}{|c|c|c|c|c|c|}
\hline Estudio & $\begin{array}{c}\text { Edad de la } \\
\text { muestra al inicio }\end{array}$ & $\begin{array}{l}\text { Marcadores } \\
\text { de riesgo }\end{array}$ & $\begin{array}{c}\text { Grupos de } \\
\text { comparación }\end{array}$ & $\begin{array}{l}\text { Diagnóstico de } \\
\text { los padres }\end{array}$ & $\begin{array}{l}\text { Intervalo de } \\
\text { seguimiento }\end{array}$ \\
\hline $\begin{array}{l}\text { John, Mednick y } \\
\text { Schulsinger } \\
\text { (1982) } \\
\text { (Copenhagen } \\
\text { High-Risk } \\
\text { Project) }\end{array}$ & $\begin{array}{l}\text { Entre } 9 \text { y } 20 \\
\text { años, media } \\
15.1\end{array}$ & $\begin{array}{l}\text { Descendencia } \\
\text { biológica de } \\
\text { madres } \\
\text { diagnosticadas } \\
\text { de SQZ grave }\end{array}$ & $\begin{array}{l}\text { (1) } 207 \\
\text { descendientes } \\
\text { biologicos de } \\
\text { madres SQZ; } \\
\text { (2) } 104 \\
\text { descendientes } \\
\text { de padres } \\
\text { mentalmente } \\
\text { sanos }\end{array}$ & $\begin{array}{l}\text { Criterios } \\
\text { DSM-III }\end{array}$ & De 1962 a 1974 \\
\hline $\begin{array}{l}\text { Watt, Grubb y } \\
\text { Erlenmeyer- } \\
\text { Kimling (1982) }\end{array}$ & $\begin{array}{l}\text { Entre } 12 \text { y } 17 \\
\text { años, media } 15\end{array}$ & $\begin{array}{l}\text { Descendencia } \\
\text { biologica de } \\
\text { uno o ambos } \\
\text { padres SQZ }\end{array}$ & $\begin{array}{l}\text { (1) } 40 \text { Hijos con } \\
\text { uno de los } \\
\text { padres afectos } \\
\text { de SQZ; (2) } 4 \\
\text { Hijos con } \\
\text { ambos padres } \\
\text { SQZ; (3) } 6 \text { Hijos } \\
\text { con uno de los } \\
\text { padres } \\
\text { psicotico- } \\
\text { depresivo; (4) } \\
70 \text { controles } \\
\text { normales }\end{array}$ & $\begin{array}{l}\text { Criterios } \\
\text { DSM-III }\end{array}$ & $\begin{array}{l}\text { De los } 12-15 \text { a } \\
\text { los } 14-17 \text { años }\end{array}$ \\
\hline $\begin{array}{l}\text { Marcus et al. } \\
\text { (1987) revisión: } \\
\text { A: The Israeli } \\
\text { Kibbutz-City } \\
\text { Study }\end{array}$ & $\begin{array}{l}\text { A: Entre } 8.1 \text { y } \\
14.8 \text { años, } \\
\text { media } 11.3\end{array}$ & $\begin{array}{l}\text { A: Descendencia } \\
\text { biologica de } \\
\text { padre o madre } \\
\text { esquizofrénicos }\end{array}$ & $\begin{array}{l}\text { A: (1) Grupo de } \\
\text { riesgo; (2) } \\
\text { Descendencia } \\
\text { biológica de } \\
\text { padres } \\
\text { mentalmente } \\
\text { sanos }\end{array}$ & $\begin{array}{l}\text { A: 1.Diagnóstico } \\
\text { clínico de SQZ, } \\
\text { 2. Diversas } \\
\text { hospitalizaciones } \\
\text { debidas a este } \\
\text { trastorno, } \\
\text { 3. Al menos tres } \\
\text { sintomas } \\
\text { psicóticos }\end{array}$ & De 1967 a 1981 \\
\hline $\begin{array}{l}\text { B: The } \\
\text { Jerusalem Infant } \\
\text { Development } \\
\text { Study }\end{array}$ & $\begin{array}{l}\text { B: } 3 \text { días } \\
\text { después de su } \\
\text { nacimiento }\end{array}$ & $\begin{array}{l}\text { B: Descendencia } \\
\text { biologica de al } \\
\text { menos uno de } \\
\text { los padres } \\
\text { esquizofrénico }\end{array}$ & $\begin{array}{l}\text { B: (1) } 19 \\
\text { descendientes } \\
\text { de SQZ; (2) } 6 \\
\text { hijos de padres } \\
\text { con tr. afectivo; } \\
\text { (3) } 14 \text { hijos de } \\
\text { padres } \\
\text { neuróticos o } \\
\text { con tr. de } \\
\text { personalidad; } \\
\text { (4) } 19 \text { hijos de } \\
\text { padres sanos }\end{array}$ & $\begin{array}{l}\text { B: Criterios } \\
\text { DSM-II y } \\
\text { DSM-III }\end{array}$ & $\begin{array}{l}\text { De los } 3 \text { días } \\
\text { después de su } \\
\text { nacimiento a } \\
\text { los } 12 \text { meses de } \\
\text { edad }\end{array}$ \\
\hline $\begin{array}{l}\text { Erlenmeyer- } \\
\text { Kimling y } \\
\text { Cornblatt (1993; } \\
\text { 1987) (The New } \\
\text { York High-Risk } \\
\text { Project) }\end{array}$ & $\begin{array}{l}\text { Muestras A y B: } \\
\text { entre } 7 \text { y } 12 \\
\text { años }\end{array}$ & $\begin{array}{l}\text { Descendecia } \\
\text { biológica de } \\
\text { padres } \\
\text { afactados por } \\
\text { trs. } \\
\text { esquizofrénicos } \\
\text { y trs. afectivos } \\
\text { mayores }\end{array}$ & $\begin{array}{l}\text { (1) Hijos de } \\
\text { padres } \\
\text { esquizofrénicos; } \\
\text { (2) Hijos de } \\
\text { padres con trs. } \\
\text { afectivos } \\
\text { mayores; } \\
\text { (3) Hijos de } \\
\text { padres sin } \\
\text { patología mental }\end{array}$ & SADS-L; RDC; & $\begin{array}{l}\text { De } 1971 \text { a } 1990 \\
\text { (A) } \\
\text { De } 1977 \text { a } 1984 \\
\text { (B: muestra de } \\
\text { replicación) }\end{array}$ \\
\hline
\end{tabular}


Tabla 1. (Continuación)

\begin{tabular}{|c|c|c|c|c|c|}
\hline Estudio & $\begin{array}{c}\text { Edad de la } \\
\text { muestra al inicio }\end{array}$ & $\begin{array}{c}\text { Marcadores } \\
\text { de riesgo }\end{array}$ & $\begin{array}{c}\text { Grupos de } \\
\text { comparación }\end{array}$ & $\begin{array}{c}\text { Diagnóstico de } \\
\text { los padres }\end{array}$ & $\begin{array}{l}\text { Intervalo de } \\
\text { seguimiento }\end{array}$ \\
\hline $\begin{array}{l}\text { Cannon, } \\
\text { Mednick y } \\
\text { Parnas (1990) } \\
\text { (Copenhagen } \\
\text { High-Risk } \\
\text { Project) }\end{array}$ & $\begin{array}{l}\text { Entre } 9 \text { y } 20 \\
\text { años, media } \\
15.1\end{array}$ & $\begin{array}{l}\text { Descendencia } \\
\text { biológica de } \\
\text { madres } \\
\text { diagnosticadas } \\
\text { de SQZ grave }\end{array}$ & $\begin{array}{l}138 \\
\text { descendientes } \\
\text { biologicos de } \\
\text { madres SQZ }\end{array}$ & $\begin{array}{l}\text { Criterios } \\
\text { DSM-III }\end{array}$ & De 1962 a 1989 \\
\hline $\begin{array}{l}\text { Parnas et al. } \\
\text { (1993) } \\
\text { (Copenhagen } \\
\text { High-Risk } \\
\text { Project) }\end{array}$ & $\begin{array}{l}\text { Entre } 9 \text { y } 20 \\
\text { años, media } \\
15.1\end{array}$ & $\begin{array}{l}\text { Descendencia } \\
\text { biológica de } \\
\text { madres } \\
\text { diagnosticadas } \\
\text { de SQZ grave }\end{array}$ & $\begin{array}{l}\text { (1) } 207 \text { sujetos } \\
\text { de alto riesgo; } \\
\text { (2) } 104 \text { sujetos } \\
\text { controles }\end{array}$ & $\begin{array}{l}\text { Criterios } \\
\text { DSM-III-R } \\
\text { (diagnósticos } \\
\text { maternos } \\
\text { revisados) }\end{array}$ & $\begin{array}{l}\text { De los } 15 \text { a los } \\
42 \text { años }\end{array}$ \\
\hline
\end{tabular}

Abreviaciones: SQZ (Esquizofrenia); SADS-L (Schedule for Affective disonders and Schizophrenia-Lifetime version: RDC (Research Dlagnostic Criteria).

infantil equiparables a las que proporcionan los maestros mediante sus informes escolares.

La validez ecológica que avala las observaciones de los maestros del comportamiento infantil responde de la calidad de los datos que aportan, recogidos en un marco estimular estable (horarios escolares, escenarios limitados - el aula, el patio...-) al tiempo que rico (en cuanto que susceptible de interacción social, juego, manifestación de capacidades...). Por esta razón, no ha de extrañar que se pueda concluir, sobre la base de estas fuentes, sobre el valor predictivo de la disarmonía de las relaciones con los compañeros, el retraimiento social, la inestabilidad e inmadurez emocionales, la impulsividad, la agresividad, la irritabilidad y la ansiedad, así como de la introversión, la desatención y la pasividad escolares, o de la inteligencia, tal y como a continuación se demuestra.

Watt y Lubensky (1976) dedujeron, de la comparación de informes escolares, que los pre-esquizofrénicos tienden al aislamiento social, a la inmadurez emocional y a un rendimiento escolar inferior a la media. Además, coinciden con John, Mednick y Schulsinger (1982) en afirmar que si bien los chicos exhiben más problemas disciplinarios y conductas anti- sociales, las chicas son manifiestamente más introvertidas, solitarias y socialmente inseguras.

Watt, Grubb y Erlenmeyer-Kimling (1982) concretaron, sobre la base de la comparación de 44 descendientes biológicos de padres esquizofrénicos y 70 hijos de padres normales, que la inestabilidad emocional, la falta de armonía en la relación con los compañeros y la baja inteligencia son los marcadores tempranos de vulnerabilidad con mayor valor predictivo. Los sujetos de riesgo se mostraban menos populares, agradables y socialmente adaptados que sus compañeros escolares, y más negativistas, nerviosos y emotivos que ellos.

En 1990, el Copenhagen High-Risk Project permitió distinguir entre antecedentes de la sintomatología positiva de la esquizofrenia y antecedentes de la sintomatología negativa. A los 15 años, los sujetos que posteriormente cursaron síntomas predominantemente negativos fueron descritos por sus educadores como pasivos, solitarios e indiferentes al elogio, mientras que a los que cursaron un cuadro sintomático predominantemente positivo se les describió como hiperactivos, irritables, agresivos y distraíbles (Cannon, Mednick y Parnas, 1990). Esta distinción no sólo corrobora que la pato- 
logía grave es precedida por alteraciones premórbidas (Chapman, 1966; Strauss, 1969), sino que extiende el valor pronóstico del trastorno a la adultez.

La fundamentación de esta propuesta se encuentra en los hallazgos de Maziade et al. (1996), que convergen con otros estudios en que tanto el funcionamiento premórbido como la gravedad de los síntomas positivos y negativos durante los episodios agudos (sobretodo la de los positivos) son buenos predictores del pronóstico a largo plazo. Parnas et al. (1993) corroboraron la agregación familiar de la esquizofrenia, hallada anteriormente por Schulsinger (1976) en la misma muestra danesa, añadiendo que entre la descendencia biológica de madres esquizofrénicas cabe distinguir un $16 \%$ de trastornos esquizofrénicos, un 4,6\% de psicosis funcionales y un $21 \%$ de trastornos esquizotípico, paranoide o esquizoide de la personalidad.

Marcus et al. (1987), por su parte, revisaron dos estudios basados en muestras israelíes que permiten contrastar los datos recién expuestos. El NIMH Israeli Kibbutz-City Study versa sobre el seguimiento de 100 preadolescentes hasta el inicio de la etapa adulta. De la comparación de los informes de maestros, padres y niños se obtuvieron los patrones conductuales distintivos de los 50 niños de alto riesgo. En este caso, los chicos presentan tendencia a la introversión y/o a la conducta antisocial, mientras que las chicas refieren síntomas más internalizantes como la retracción social o el sentimiento de no pertenencia a un grupo.

El segundo estudio que recogen (The Jerusalem Infant Development Study), evalúa el desarrollo perceptivo, motor y atencional de 58 niños descendientes de padres afectados por trastornos afectivos, de personalidad o esquizofrénicos. Mediante la aplicación de la escala de Brazelton a los pocos días del nacimiento, y la escala de Bayley a los 4, 8 y 12 meses, constataron la existencia de déficit motores y sensorio-motrices en el primer año de vida de 13 de los 19 descendientes biológicos de padres esquizofrénicos.

El mismo año, Erlenmeyer-Kimling y Cornblatt (1987) publican resultados del proyecto de alto riesgo de Nueva York, en el que los marcadores bioconductuales son el funcionamiento neuromotor, la capacidad atencional y la respuesta electrodermal. Mediante un índice atencional compuesto, que integra resultados de tres pruebas (Continuous Perfomance Test, Auditory Attention Span Task, y las pruebas de Dígitos directos e inversos del WISC), constatan la presencia de déficit atencionales en los sujetos de riesgo, cuya motricidad refleja además graves alteraciones neurológicas, pero no consiguen demostrar la validez de la respuesta galvánica como indicador temprano de vulnerabilidad genética a la esquizofrenia, a diferencia de otros grupos que habian trabajado exitosamente con este marcador (como, por ejemplo, el grupo danés).

En 1993 las mismas autoras completan los hallazgos añadiendo la relación entre el índice atencional compuesto (ADI: Attention Deviance Index) y la anhedonia, relación estadísticamente significativa sólo en el grupo de riesgo de esquizofrenia. Además, aducen que aunque los hombres manifiestan niveles más elevados de incapacidad para sentir placer, sólo en las mujeres la anhedonia marca realmente riesgo de psicosis (ErlenmeyerKimling et al., 1993).

Hay que reconocer el valor de todas estas aportaciones sin ignorar las dificultades metodológicas que asumen los autores en el curso de sus estudios. Son muchos los factores que pueden distorsionar los resultados, tales como el tamaño de las muestras, que cuánto más reducido más dificulta el proceso de generalización, o el hecho de que, en 
algunos casos, las hipótesis de partida se fundamenten en análisis previos de los mismos datos. Así, el rigor que el lector atribuya a las conclusiones de cada estudio debería contemplar las limitaciones del mismo.

\section{PRECURSORES CONDUCTUALES INFANTILES DE LA ESQUIZOTIPIA}

Diferentes estudios confirman la relación etiológica entre la esquizofrenia y el trastorno esquizotípico de la personalidad (Ritsner, Karas y Ginath, 1993; Torgersen, Onstad, Skre, Edvarsen y Kringlen, 1993). La complejidad de esta relación sugiere no limitar la intersección entre esquizofrenia y esquizotipia al trastorno esquizotípico de la personalidad propiamente dicho, sino que invita a ampliarla a los ragos esquizotípicos presentes en cuadros subclínicos.
Debe distinguirse entre cuatro posibilidades de relación entre esquizofrenia y personalidad. 1) En primer lugar, puede que rasgos de personalidad atípicos precedan al desarrollo de un episodio esquizofrénico agudo. 2) Puede que la sintomatología esquizofrénica sea concominante a características no psicóticas de la personalidad. 3) Es posible que un trastorno específico de la personalidad suceda a un cuadro agudo de esquizofrenia y, finalmente, 4) también cabe que una constelación de rasgos surja como alternativa a un cuadro esquizofrénico, partiendo de los mismos factores etiológicos (Torgersen, 1994).

Esta última posibilidad es la que ampara el sentido de las desviaciones de la personalidad como una entidad diferente dentro del espectro de la esquizofrenia. A continuación se describen los hallazgos de los principales estudios del tema y pueden encontrarse los aspectos metodológicos en la tabla 2.

Tabla 2. Selección de estudios sobre precursores de esquizotipia

\begin{tabular}{|c|c|c|c|c|}
\hline Estudio & Diseño/Muestra & Objetivos/Hipotesis & Instrumentos & Resultados \\
\hline Wolff et al. (1991) & $\begin{array}{l}\text { Diagnóstico y } \\
\text { seguimiento } \\
\text { durante } 24 \text { años de } \\
32 \text { niños } \\
\text { «esquizoides» en } \\
\text { su infancia y } \\
\text { comparación con } \\
32 \text { niños controles }\end{array}$ & $\begin{array}{l}\text { Demostrar que los } \\
\text { pacientes infantiles } \\
\text { «esquizoides» son } \\
\text { fenomenológicame } \\
\text { nte similares, } \\
\text { en la adultez, a los } \\
\text { sujetos con tr. del } \\
\text { espectro } \\
\text { esquizofréntco }\end{array}$ & $\begin{array}{l}\text { Mill Hill } \\
\text { Vocabulary Test, } \\
\text { Matrices de Raven; } \\
\text { Baron Schedule for } \\
\text { Schizotypal } \\
\text { Personality } \\
\text { Disorder, SADS-L; } \\
\text { Diagnostic Interview } \\
\text { for Borderlines, } \\
\text { DSM-III }\end{array}$ & $\begin{array}{l}24 \text { de los } 32 \text { sujetos } \\
\text { diagnosticados de } \\
\text { «esquizoides» } \\
\text { en su infancia } \\
\text { cumplen criterios } \\
\text { DSM-III para el } \\
\text { diagnóstico SPD en } \\
\text { la adultez }\end{array}$ \\
\hline Lencz et al. (1993) & $\begin{array}{l}\text { Comparación de los } \\
\text { déficit en el } \\
\text { seguimiento ocular } \\
\text { de } 14 \text { sujetos SPD } \\
\text { (DSM-III-R) y } 18 \\
\text { controles ( } n=32, \\
\text { edad media } 19 \text { años) }\end{array}$ & $\begin{array}{l}\text { Los universitarios } \\
\text { seleccionados por } \\
\text { su deficiencia en el } \\
\text { seguimiento ocular } \\
\text { presentaran mayor } \\
\text { prevalencia de SPD } \\
\text { que los controles }\end{array}$ & $\begin{array}{l}\text { SPQ; WAIS-R; } \\
\text { Registro de los } \\
\text { movimientos } \\
\text { oculares; DSM-III-R }\end{array}$ & $\begin{array}{l}\text { El grupo SPD } \\
\text { presenta mayores } \\
\text { déficit en el } \\
\text { seguimiento ocular } \\
\text { que el grupo } \\
\text { control }\end{array}$ \\
\hline Torgersen (1994) & $\begin{array}{l}\text { Revisión de } \\
\text { estudios de } \\
\text { familias, gemelos y } \\
\text { adopciones. }\end{array}$ & $\begin{array}{l}\text { Descubrir qué rasgos } \\
\text { de personalidad no } \\
\text { psicótica tienen el } \\
\text { mismo origen que la } \\
\text { SQZ y pueden ser } \\
\text { una alternativa no- } \\
\text { psicótica a esta } \\
\text { enfermedad }\end{array}$ & $\cdot$ & $\begin{array}{l}\text { Sólo el SPD parece } \\
\text { estar } \\
\text { etiológicamente } \\
\text { ligado a la SQZ }\end{array}$ \\
\hline
\end{tabular}


Tabla 2. (Continuación)

\begin{tabular}{|c|c|c|c|c|}
\hline Estudio & Diseño/Muestra & Objetivos/Hipótesis & Instrumentos & Resultados \\
\hline $\begin{array}{l}\text { Chapman et al. } \\
\text { (1994) }\end{array}$ & $\begin{array}{l}\text { Administración del } \\
\text { conjunto de escalas } \\
\text { de "psychosis- } \\
\text { proneness" a } 7800 \\
\text { universita-rios. } \\
\text { Seguimiento } \\
\text { prospectivo } \\
\text { durante } 10 \text { años. }\end{array}$ & $\begin{array}{l}\text { Análisis del valor } \\
\text { predictivo de las } \\
\text { diversas escalas } \\
\text { para el desarrollo } \\
\text { de trastornos } \\
\text { psicóticos. }\end{array}$ & $\begin{array}{l}\text { Perceptual } \\
\text { Aberration Scale; } \\
\text { Anhedonia física, } \\
\text { Anhedonia social, } \\
\text { Ideación mágica e } \\
\text { Impulsividad no } \\
\text { conformista. }\end{array}$ & $\begin{array}{l}\text { Las elevadas } \\
\text { puntuaciones de } \\
\text { PAB, ideación } \\
\text { mágica y anhedonia } \\
\text { social son índices } \\
\text { pronósticos válidos } \\
\text { de diagnóstico de } \\
\text { psicosis y } \\
\text { esquizotipia. }\end{array}$ \\
\hline $\begin{array}{l}\text { Erlenmeyer- } \\
\text { Kimling (1995) }\end{array}$ & $\begin{array}{l}\text { Prospectivo de } \\
\text { seguimiento de } 188 \\
\text { hijos de padres con } \\
\text { SQZ, tr. afectivo y } \\
\text { controles de los } 7- \\
12 \text { años (1971) a los } \\
30(1994)\end{array}$ & $\begin{array}{l}\text { Estudiar la } \\
\text { agregación familiar } \\
\text { de psicosis y } \\
\text { trastornos de la } \\
\text { personalidad del } \\
\text { tipo A según } \\
\text { criterios DSM-III-R }\end{array}$ & $\begin{array}{l}\text { SADS-L; PDE; } \\
\text { MMPI; DSM-III-R }\end{array}$ & $\begin{array}{l}\text { La prevalencia de } \\
\text { la esquizofrenia es } \\
\text { de } 11.1 \% \pm 3.4 \% \\
\text { en el grupo de } \\
\text { riesgo, y } 0 \% \text { en } \\
\text { descendientes de } \\
\text { padres con } \\
\text { trastornos afectivos } \\
\text { y controles }\end{array}$ \\
\hline Tyrka et al. (1995) & $\begin{array}{l}\text { Diagnóstico según } \\
\text { DSM-III-R de los } \\
207 \text { niños de riesgo } \\
\text { y los } 104 \text { controles } \\
\text { que conforman la } \\
\text { Copenhagen High- } \\
\text { Risk sample }\end{array}$ & $\begin{array}{l}\text { Evaluar los } \\
\text { indicadores } \\
\text { premórbidos de } \\
\text { patología del } \\
\text { espectro } \\
\text { esquizofrénico }\end{array}$ & $\begin{array}{l}\text { PSE; SADS-L; } \\
\text { DSM-III-R }\end{array}$ & $\begin{array}{l}\text { La agregación de } \\
\text { los indicadores es } \\
\text { consistente con } \\
\text { una distribución } \\
\text { bimodal de la } \\
\text { vulnerabilidad } \\
\text { latente }\end{array}$ \\
\hline Gruzelier (1996) & $\begin{array}{l}\text { Evaluación de rasgos } \\
\text { esquizotípicos de } \\
\text { 151 estudiantes de } \\
\text { medicina ( } 73 \\
\text { chicos,78 chicas) } \\
\text { divididos } \\
\text { alfabéticamente en } \\
\text { dos grupos }\end{array}$ & $\begin{array}{l}\text { Replicar la } \\
\text { validación de la } \\
\text { estructura } \\
\text { trifactorial de la } \\
\text { esquizotipia } \\
\text { medida mediante } \\
\text { el SPQ }\end{array}$ & SPQ; PAS & $\begin{array}{l}\text { Evidencia } \\
\text { confirmatoria de la } \\
\text { estructura } \\
\text { trifactorial del } \\
\text { constructo } \\
\text { esquizotípico }\end{array}$ \\
\hline $\begin{array}{l}\text { Altman, Collins y } \\
\text { Mundy (1997) }\end{array}$ & $\begin{array}{l}21 \text { chicos y } 17 \\
\text { chicas de entre } 13 \\
\text { y } 21 \text { años (media } \\
\text { 16) se presentan } \\
\text { voluntariamente a } \\
\text { la evaluación }\end{array}$ & $\begin{array}{l}\text { Predicciones: las } \\
\text { alucinaciones y/o } \\
\text { delirios subclínicos } \\
\text { se relacionan con } \\
\text { 1) cuadros } \\
\text { disociativos, 2) } \\
\text { procesos } \\
\text { esquizotípicos de } \\
\text { pensamiento y 3) } \\
\text { trasfondos } \\
\text { depresivos }\end{array}$ & $\begin{array}{l}\text { CI verbal } \\
\text { (Peabody); } \\
\text { sintomatología } \\
\text { (Rust Inventory of } \\
\text { Schizotypal } \\
\text { Thinking, The } \\
\text { Dissociative } \\
\text { Experiences Scale, } \\
\text { CDI, Diagnostic } \\
\text { Inventory } \\
\text { Schedule) }\end{array}$ & $\begin{array}{l}\text { Tanto las } \\
\text { experiencias } \\
\text { disociativas, la } \\
\text { sintomatología } \\
\text { depresiva, y los } \\
\text { procesos } \\
\text { esquizotípicos de } \\
\text { pensamiento se } \\
\text { asocian a las } \\
\text { alucinaciones } \\
\text { auditivas y/o } \\
\text { delirios subclínicos }\end{array}$ \\
\hline $\begin{array}{l}\text { Obiols et al. (1997) } \\
\text { y Obiols et al. (en } \\
\text { prensa) }\end{array}$ & $\begin{array}{l}1498 \text { adolescentes } \\
\text { de la población } \\
\text { general (media } 13 \\
\text { años) son cribados } \\
\text { mediante el CPT, } \\
\text { formándose un } \\
\text { grupo de bajo } \\
\text { rendimiento } \\
\text { atencional (índice) } \\
\text { y uno control }\end{array}$ & $\begin{array}{l}\text { El déficit } \\
\text { atencional en } \\
\text { población } \\
\text { adolescente sana se } \\
\text { asociará a } \\
\text { presencia de otros } \\
\text { marcadores de } \\
\text { riesgo y será } \\
\text { predicitivo de } \\
\text { futura patología del } \\
\text { espectro sqz sin } \\
\text { presencia de riesgo } \\
\text { genético }\end{array}$ & $\begin{array}{l}\text { Raven; PAB; PAS; } \\
\text { Escala de } \\
\text { Anhedonia Social; } \\
\text { Wisconsin Card } \\
\text { Sorting Test; Trail } \\
\text { Making Tests; FAS; } \\
\text { Achenbach } \\
\text { Teachers Report } \\
\text { Form; Batería de } \\
\text { signos neurologicos } \\
\text { menores; } \\
\text { Edinburgh } \\
\text { Laterality Scale }\end{array}$ & $\begin{array}{l}\text { Los sujetos índice } \\
\text { presentan menor } \\
\text { inteligencia, peor } \\
\text { función ejecutiva, } \\
\text { más signos } \\
\text { esquizotípcos } \\
\text { negativos, más } \\
\text { signos neurológicos } \\
\text { menores, mayor } \\
\text { proporición de } \\
\text { zurdos y más } \\
\text { problemas sociales } \\
\text { informados por los } \\
\text { maestros respecto } \\
\text { al grupo control }\end{array}$ \\
\hline
\end{tabular}


Tabla 2. (Continuación)

\begin{tabular}{|c|c|c|c|c|}
\hline Estudio & Diseño/Muestra & Objetivos/Hipótesis & Instrumentos & Resultados \\
\hline Olin et al. (1997) & $\begin{array}{l}\text { Comparación de } \\
\text { los informes } \\
\text { escolares } 36 \text { sujetos } \\
\text { SPD (DSM-III-R), } \\
31 \text { SQZ y } 165 \\
\text { sujetos } \\
\text { diagnosticados de } \\
\text { patologías distintas } \\
\text { o sanos } \\
\text { (Copenhagen High- } \\
\text { Risk sample) }\end{array}$ & $\begin{array}{l}\text { a) los sujetos SPD } \\
\text { serán más } \\
\text { introvertidos en su } \\
\text { infancia y } \\
\text { presentarán } \\
\text { problemas } \\
\text { externalizantes } \\
\text { iguales a los de los } \\
\text { no psicóticos, b) } \\
\text { presentaran iguales } \\
\text { problemas } \\
\text { internalizantes y } \\
\text { menores } \\
\text { externalizantes que } \\
\text { los SQZ }\end{array}$ & $\begin{array}{l}\text { PSE; PSE Etiology } \\
\text { Scale; PSE } \\
\text { syndrome } \\
\text { Checklist; PDE; } \\
\text { SPQ; DSM-III-R; } \\
\text { DSM-IV }\end{array}$ & $\begin{array}{l}\text { Los sujetos } \\
\text { esquizotípicos se } \\
\text { presentan más } \\
\text { pasivos e } \\
\text { hipersensibles a la } \\
\text { crítica en su } \\
\text { infancia que los } \\
\text { esquizofrénicos }\end{array}$ \\
\hline $\begin{array}{l}\text { Chen, Hsiao y } \\
\text { Chaucer (1997) }\end{array}$ & $\begin{array}{l}\text { Evaluación rasgos } \\
\text { esquizotípicos de } \\
345 \text { adultos y } 115 \\
\text { adolescentes } \\
\text { taiwaneses }\end{array}$ & $\begin{array}{l}\text { Verificar si la } \\
\text { estrucutra } \\
\text { trifactorial del } \\
\text { SPQ se presenta } \\
\text { en ambas } \\
\text { muestras y si el } \\
\text { factor } \\
\text { interpersonal se } \\
\text { asocia con la } \\
\text { desatención }\end{array}$ & SPQ; PAB; CPT & $\begin{array}{l}\text { Los rasgos } \\
\text { esquizotípicos } \\
\text { correlacionan más } \\
\text { en la adolescencia } \\
\text { ( } 2 \text { factores) y se } \\
\text { diferencian al } \\
\text { llegar a la etapa } \\
\text { adulta }\end{array}$ \\
\hline Kwapil (1998) & $\begin{array}{l}\text { Comparación y } \\
\text { seguimiento } \\
\text { durante } 10 \text { años, } \\
\text { de } 34 \\
\text { universitarios ( } 20 \\
\text { años) con altos } \\
\text { indices de } \\
\text { anhedonia y } 139 \\
\text { sujetos control }\end{array}$ & $\begin{array}{l}\text { Evaluar el valor de } \\
\text { la anhedonia como } \\
\text { predictor de } \\
\text { patología del } \\
\text { espectro } \\
\text { esquizofrénico }\end{array}$ & $\begin{array}{l}\text { PDE; GAS; MI; } \\
\text { PAB; SAS; Social } \\
\text { Adjustment Scale; } \\
\text { DSM-IV }\end{array}$ & $\begin{array}{l}\text { El grupo } \\
\text { anhedónico supera } \\
\text { al control en } \\
\text { gravedad de } \\
\text { patología psicótica } \\
\text { y ajuste global al } \\
\text { final del } \\
\text { seguimiento ( } 30 \\
\text { años) }\end{array}$ \\
\hline $\begin{array}{l}\text { O'Driscoll, } \\
\text { Lenzenweger y } \\
\text { Holzman (1998) }\end{array}$ & $\begin{array}{l}\text { Evaluación del } \\
\text { seguimiento ocular } \\
\text { y los movimientos } \\
\text { antisacádicos en } 31 \\
\text { universitarios con } \\
\text { alta percepción } \\
\text { aberrantes y } 24 \text { con } \\
\text { baja percepción } \\
\text { aberrante }\end{array}$ & $\begin{array}{l}\text { El grupo con rasgos } \\
\text { esquizotipicos } \\
\text { tendrá un } \\
\text { rendimiento más } \\
\text { pobre que el } \\
\text { control en ambas } \\
\text { pruebas }\end{array}$ & $\begin{array}{l}\text { PAB; BDI; } \\
\text { Diagnostic } \\
\text { Interview } \\
\text { Schedule: } \\
\text { Scholastic } \\
\text { Aptitude Test; } \\
\text { Evaluación del } \\
\text { seguimiento ocular } \\
\text { y los movimientos } \\
\text { antisacádicos }\end{array}$ & $\begin{array}{l}\text { Se cumplen las } \\
\text { predicciones, y los } \\
\text { errores más } \\
\text { comunes } \\
\text { cometidos por el } \\
\text { grupo } \\
\text { esquizotipico son } \\
\text { las perseveraciones }\end{array}$ \\
\hline $\begin{array}{l}\text { Wolfradt y Straube } \\
\text { (1998) }\end{array}$ & $\begin{array}{l}\text { Se evalúa la } \\
\text { sintomatologia } \\
\text { esquizotípica, } \\
\text { ansiosa y depresiva } \\
\text { de } 1362 \\
\text { estudiantes de } 15 \\
\text { años }\end{array}$ & $\begin{array}{l}\text { Evaluar la } \\
\text { estructura factorial } \\
\text { de los rasgos de } \\
\text { personalidad } \\
\text { esquizotípica en } \\
\text { adolescentes (14- } \\
\text { 18años) }\end{array}$ & $\begin{array}{l}\text { STA: Freiburg } \\
\text { Personality } \\
\text { Inventory } \\
\text { (subscales: anxiety } \\
\text { (N) and } \\
\text { depressivity (D)) }\end{array}$ & $\begin{array}{l}\text { Por separado, las } \\
\text { matrices de } \\
\text { correlaciones de } \\
\text { ambos sexos } \\
\text { corroboran la } \\
\text { trifactorialidad, } \\
\text { pero } \\
\text { conjuntamente } \\
\text { sugieren } 4 \text { factores } \\
\text { estructurales }\end{array}$ \\
\hline
\end{tabular}


Tabla 2. (Continuación)

\begin{tabular}{|c|c|}
\hline Estudio & Diseño/Muestra \\
\hline $\begin{array}{l}\text { Kravetz, Faust y } \\
\text { Edelman (1998) }\end{array}$ & $\begin{array}{l}\text { Análisis del tiempo } \\
\text { de reacción y la } \\
\text { precisión en una } \\
\text { tarea de decisión } \\
\text { léxica de } 60 \\
\text { universitarios } \\
\text { (chicos) separados } \\
\text { en } 3 \text { grupos según } \\
\text { sus características } \\
\text { esquizotípicas }\end{array}$ \\
\hline
\end{tabular}

Chen et al. (1998)

Moritz, Mass y

Junk (1998)

Cassady et al. (1998)

\section{Evaluación de la atención sostenida en 60 esquizofrénicos, 148 de sus familiares de 1 er grado, 20 sujetos control y 42 de sus familiares de 1er grado}

Evaluación del negative priming en 100 sujetos sanos

\begin{tabular}{|c|c|}
\hline $\begin{array}{l}\text { Evaluación de los } \\
\text { movimientos } \\
\text { discinéticos en } 22 \\
\text { sujetos normales y } \\
34 \text { con } \\
\text { personalidades del } \\
\text { espectro } \\
\text { esquizofrénico }\end{array}$ & $\begin{array}{l}\text { Hipotetizan que los } \\
\text { sujetos con } \\
\text { personalidades del } \\
\text { espectro } \\
\text { esquizofrénico } \\
\text { (esquizoide, } \\
\text { paranoide o } \\
\text { esquizotípica) } \\
\text { presentan } \\
\text { discinesia } \\
\text { espontánea }\end{array}$ \\
\hline
\end{tabular}

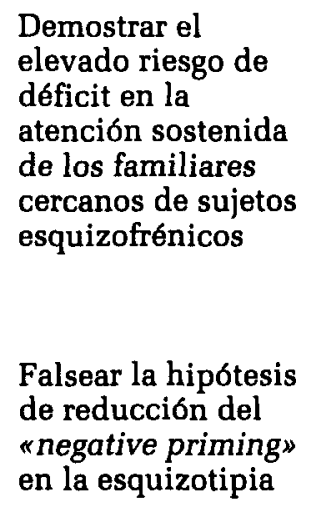

Demostrar el elevado riesgo de déficit en la atención sostenida de los familiares cercanos de sujetos esquizofrénicos

Falsear la hipótesis de reducción del "negative priming" en la esquizotipia
CPT; Chinese version of Diagnostic Intervew for Genetic Studies (DIGS-C); DSM-III- $\mathrm{R}$ O-LIFE; tarea de decisión léxica

Menor efectividad en el procesamiento lingüístico para las dimensiones "unusual experiences" $\mathrm{y}$ "cognitive disorganisation” (HI). La mayor efectividad es en "impulsive nonconformity" (HD). PAB; SPQ; DSM-IV

Los valores de d' en familiares de SQZ son inferiores a los de familiares de controles y mayores que de pacientes SQZ
Las alteraciones perceptivas no contribuyen a reducir este efecto en sujetos altamente esquizotípicos

Presencia de discinesia en el $24 \%$ de los esquizotípicos pero no en los otros grupos.

Abreviaciones: BDI (Beck Depression Inventory); CI (Cociente de inteligencia); CDI (Child Depression Inventory); CPT (Continuous Perfomance Test); GAS (Global Adjustment Scale); MI (Magical Ideation Scale); MMPI (Minnesota Multiphasic Personality Inventory); O-LIFE (Oxford-Liverpool Inventory of Feelings and Experiences); PAB (Perceptual Aberration Scale); PAS (Physical Anhedonia Scale); PDE (Personality Disorder Exam); PSE (Present State Examination); SADS-L (Shedule for Affective Disorders and SQZ-Lifetime version); SPD (Schizotypal Personality Disorder); SPQ (Schizotypal Personality Questionnaire); STA (Schizotypal Personality Scale); SQZ (Schizophrenia); WAIS-R (Wechsler Adult Intelligence Scale-Revised).

Wolff (1991) describió síntomas esquizoides en 32 pacientes menores de 10 años cuyo seguimiento hasta los 27 permitió diagnosticar trastorno esquizotípico de la personalidad (DSM-III) en 24 de ellos. Distinguió a los sujetos que él denomina "esquizoides» mediante el Baron Schedule for Schyzotypal Personality Disorder, escala especialmente sensible a la detección de ideas de refe- 
rencia, pensamiento mágico, aislamiento social y, sobretodo, dificultades de relación y comunicación. Hay que tener en cuenta que sólo 6 de los 32 sujetos clasificados por esta escala en el grupo control, reunieron los criterios para el diagnóstico clínico de la personalidad esquizotípica. Éste es un punto a favor de la validez predictiva y de constructo de la escala.

Erlenmeyer-Kimling et al. (1995), por su parte, ilustran la agregación familiar de los trastornos de personalidad del grupo A del DSM en descendientes de esquizofrénicos aunque, si bien diversos estudios refieren la presencia específica del trastorno esquizotípico de la personalidad (Parnas et al. 1993; Maier, Lichtermann, Mingues y Heun, 1994), su trabajo revela la mayor prevalencia de los trastornos del grupo A (esquizotípico, paranoide y esquizoide) en conjunto. Algunos investigadores (como por ejemplo Siever, Keefe y Bernstein, 1991), observando la prevalencia del trastorno esquizotípico entre los familiares de pacientes no esquizofrénicos (Yeung, Lyons, Waternaux, Faraone y Tsuang, 1993), concluyen que no todos los casos de esquizotipia se encuentran genéticamente ligados a la esquizofrenia, con lo que sugieren que la relación personalidad esquizotípica-espectro esquizofrénico, en términos de la teoría de conjuntos, tiene un carácter intersectivo, ya que aunque ambos conceptos comparten rasgos, ninguno de los dos contiene enteramente al otro.

A continuación se revisarán tres áreas que cuentan con un número suficiente de estudios para permitir la elaboración de algunas conclusiones: alteraciones psicopatológicas premórbidas, función atencional y factores ambientales de tipo familiar. Dentro de los estudios de precursores cognitivos hemos seleccionado deliberadamente la función atencional dado que ha sido sin duda la más estudiada y posee además un marco teórico que hace sus resultados especialmente interesantes. $\mathrm{El}$ lector interesado en una revisión global y actualizada de los marcadores cognitivos puede referirse por ejemplo a Martínez. Lemos y Bobes (1999).

Precursores psicológicos: alteraciones psicopatologicas premórbidas

Aunque Meehl (1989) señaló que los indicadores neurológicos y psicofisiológicos están más ligados a la cadena causal de la esquizotaxia que los procesos cognitivos y sociales, ha sido claramente demostrado que el pensamiento, la imaginación y la percepción alterados son precursores de patología psicótica.

Eckblad y Chapman (1983) distiguieron dos grupos de sujetos según sus puntuaciones en la escala de Ideación Mágica, hallándose al menos 2 de las 5 características esquizotípicas que evaluaron (despersonalización, transmisión del pensamiento, ideación paranoide, comunicación extraña y aíslamiento social) en 11 de los 28 sujetos con altas puntuaciones, mientras que sólo uno de los 28 sujetos controles manifestó rasgos esquizotípicos.

El estudio más importante dentro de la estrategia de alto riesgo psicométrico es el desarrollado por el grupo de Wisconsin utilizando las diversas escalas que han desarrollado para la detección de rasgos de vulnerabilidad a la psicosis (psychosisproneness scales). En su estudio longitudinal prospectivo de 10 años de seguimiento (Chapman, Chapman, Kwapil, Eckblad y Zinfer, 1994) de 508 estudiantes universitarios, se determinó la validez predictiva de sus intrumentos para rasgos esquizotípicos y esquizofrenia. Tal y como se esperaba, los sujetos con altas puntuaciones en las escalas de Percepción Aberrante e Ideación Mágica, o en ambas, y dentro de estos aquellos individuos que informaron ya al inicio del estudio de 
experiencas de tipo psicótico, presentaban un mayor número de trastornos psicóticos que los sujetos controles al cabo del seguimiento, así como más familiares psicóticos, síntomas esquizotípicos y experiencias de tipo psicótico. Los sujetos con elevadas puntuaciones iniciales en Ideación Mágica y que además puntuaban por encima de la media en Anhedonia Social fueron los más patológicos. En cambio, las escalas de Anhedonia Física y de Impulsividad No Conformista no se mostraron efectivas en la predicción de la vulnerabilidad a la psicosis.

Posteriormente, en un reanálisis de los datos de este mismo proyecto, Kwapil (1998) encuentra que, al cabo de los 10 años de seguimiento, un $44 \%$ de los 34 sujetos clasificados con alta anhedonia social (como mínimo 1.96 DE por encima de la media) son diagnosticados de trastornos del espectro esquizofrénico frente al $1 \%$ de los sujetos del grupo control, a pesar de que en las evaluaciones iniciales no se daba tal diferencia en cuanto a la proporción de estos diagnósticos. Esto demuestra que la escala de Anhedonia Social permite identificar un alto porcentaje de individuos con riesgo de desarrollar un trastrono del espectro esquizofrénico en el futuro.

Tompson, Asarnow, Hamilton, Newell y Goldstein (1997) hallan que en una muestra de 56 niños de entre 7 y 14 años, los afectados de trastorno esquizotípico cursan con mayores niveles de trastornos del pensamiento que depresivos y controles.

Altman, Collins y Mundy (1997), tras corroborar la existencia de alucinaciones auditivas en el $33 \%$ de su muestra de adolescentes y de ideas paranoides y autorreferenciales en el $24 \%$, verifican la asociación de las alucinaciones con procesos disociativos y la de los delirios con procesos de pensamiento típicamente esquizotípico.

Finalmente, señalar el aporte de Cassady, Adami, Moran, Kunkel y Thaker
(1998) sobre la mayor frecuencia de discinesias espontáneas en la personalidad del espectro esquizofrénico. Concretamente, el $24 \%$ de los sujetos esquizotípicos de su muestra $(n=56)$ exhibía movimientos discinéticos o discinetiformes, relacionados, al parecer, con los síntomas positivos.

\section{Precursores psicológicos: capacidades atencionales}

Dentro de las pruebas atencionales con mayor complejidad metodológica y robustez se halla la evaluación del seguimiento ocular fino (SPEM o smooth pursuit eye movements). Según Siever et al. (1990), los déficit en el movimiento ocular se encuentran presentes en más del $86 \%$ de los sujetos esquizofrénicos, y su prevalencia también alcanza cotas altas en los familiares cercanos de los afectados, aunque estos resultados no han podido ser replicados por Thaker, Moran, Cassady, Adami y Tamminga (1991).

Lencz et al. (1993) intentaron verificar si este marcador permite cribar el trastorno esquizotípico de la personalidad en una muestra de 822 estudiantes, hallando peores resultados en las electro-oculografías de los sujetos diagnosticados como esquizotípicos mediante el DSMIII-R.

Recientemente, O'Driscoll, Lenzenweger y Holzman (1998) evaluaron el seguimiento ocular de 55 estudiantes universitarios con elevadas puntuaciones en la Escala de Percepción Aberrante de los 1684 evaluados, hallando que 31 de ellos obtienen peores resultados (tanto en la calidad de la actividad ocular como en el porcentaje correcto de movimientos antisacádicos) que los 24 controles, y que sus errores antisacádicos son principalmente de tipo perseverativo.

Otro de los paradigmas atencionales más utilizado ha sido el de la atención 
sostenida, y dentro de éste la prueba más utilizada ha sido el CPT (Continuous Perfomance Test). Nuestro equipo ha trabajado con esta prueba en un estudio longitudinal prospectivo de adolescentes de la población general que está siendo realizado desde 1993 en la Universidad Autónoma de Barcelona. El CPT, en su versión de pares idénticos, ha sido utilizado como marcador de vulnerabilidad neurocognitiva a los trastornos del espectro esquizofrénico. Tras un primer cribaje inicial de 1498 adolescentes de las aulas de octavo de EGB seleccionadas al azar mediante el CPT, se seleccionaron un grupo índice (bajo rendimiento atencional) y un grupo control (apareado por edad, sexo y aula), a los que evaluamos en diversas etapas y pretendemos seguir contactando en el futuro. Nuestros datos confirman la existencia de una asociación de diversos marcadores cognitivos y de marcadores de otras áreas de funcionamiento (motor, social, rendimiento escolar...) en los adolescentes sanos que presentan un bajo rendimiento en la prueba de atención sostenida en comparación a sujetos controles. Asi, los adolescentes con déficit atencional tienen tendencia a presentar más rasgos esquizotípicos, más signos neurológicos menores, menor inteligencia general y peor rendimiento en tareas ejecutivas como la categorización y la fluidez verbal (Obiols et al., 1997; Obiols, Barrantes, Serrano, Caparrós y Subirá, en prensa).

Recientemente, Chen et al. (1998) ratificaron la utilidad de las pruebas de atención para marcar la vulnerabilidad genética a los distintos fenotipos del espectro esquizofrénico. Su hipótesis es que los familiares no psicóticos de sujetos esquizofrénicos tendrán mayor riesgo de presentar déficit en la atención sostenida medida mediante el CPT. Sus resultados reflejan que los valores $d^{\prime}$ de los familiares de afectados son inferiores a los de los familiares de los controles, pero mayores que los de sus familiares esquizofrénicos.

En cuanto a los efectos de la esquizotipia sobre la capacidad para inhibir el procesamiento de los estímulos irrelevantes, existe evidencia de una menor inhibición cognitiva (es decir, más negative priming) en sujetos con elevadas puntuaciones en rasgos esquizotípicos positivos. Diversos trabajos del grupo de Oxford han arrojado datos en este sentido (revisión de estos estudios en Williams y Beech, 1997).

Moritz, Mass y Junk (1998) añadieron validez convergente y discriminante a la hipótesis de que la reducida capacidad para inhibir los estímulos irrelevantes es un correlato neurofisiológico de la esquizotipia positiva. A excepción de la subescala «ansiedad social» del SPQ, ninguna medida de la esquizotipia negativa o de la depresión se presenta asociada a esta capacidad, concluyéndose que sólo los rasgos esquizotípicos positivos son los que contribuyen a una reducción del efecto de negative priming, lo cual es interpretado como un menor grado de inhibición cognitiva.

\section{Precursores ambientales: familia y actitudes parentales}

Se revisan a continuación algunos de los trabajos que se centran en el estudio del funcionamiento familiar general y de las actitudes parentales concretamente, en los cuales hallamos en algunos casos términos diferentes para conceptos sinónimos. En otros se utilizan nombres que operacionalizan el entendimiento de las variables estudiadas, como por ejemplo es el caso del constructo de «emoción expresada». Para una mejor valoración de los trabajos el lector puede consultar el listado de instrumentos y otras consideraciones metodológicas que se hallan en la tabla 3. 
Tabla 3. Estudios sobre el ambiente familiar: Características generals

\begin{tabular}{|c|c|c|}
\hline Estudio & Diseño del estudio & Objetivos/Hipótesis \\
\hline Burman et al. (1987) & $\begin{array}{l}\text { Comparan la } \\
\text { percepcion de las } \\
\text { relaciones } \\
\text { familiares entre } \\
\text { grupos de alto y } \\
\text { bajo riesgo: } 17 \\
\text { descendientes de } \\
\text { SQZ, } 27 \text { de padres } \\
\text { con SPD, } 23 \\
\text { controles }\end{array}$ & $\begin{array}{l}\text { Predicciones: las } \\
\text { percepciones } \\
\text { premórbidas de las } \\
\text { relaciones familiares } \\
\text { son signif. peores en } \\
\text { los que desarrollan } \\
\text { SQZ que en los SPD } \\
\text { o los que, aun } \\
\text { siendo de riesgo, no } \\
\text { cursan patología de } \\
\text { adultos }\end{array}$ \\
\hline Fish (1987) & $\begin{array}{l}\text { Seguimiento de } 12 \\
\text { hijos de } \\
\text { esquizofrénicas } \\
\text { crónicas y } 12 \\
\text { controles desde las } \\
12-18 \text { horas de } \\
\text { nacer hasta los 26- } \\
27 \text { años }\end{array}$ & $\begin{array}{l}\text { Verificar si trs. } \\
\text { neurointegrativos } \\
\text { especificos } \\
\text { (pandysmaturatio } \\
\text {-PDM) marcan } \\
\text { vulnerabilidad a l } \\
\text { SQZ o al SPD }\end{array}$ \\
\hline
\end{tabular}

Goodman (1987)

Evaluación, hasta los 5 años de edad, del funcionamiento social, intelectual y neuropsiquiátrico de los hijos de 71 mujeres SQZ, 36 depresivas y 38 sanas

1) Describir las diferencias de los sjs. de riesgo;

2) Describir las cticas. del ambiente de crianza de los sjs. de riesgo;

3) Analizar las relaciones madreambiente-niño de los grupos de riesgo
McNeil y Kaij (1987)
Evaluación de los 2 Estudio de las a los 6 años, de la descendencia biológica de madres con cuadros psicóticos cticas. de la descendencia , ambiente de crianza e interacción madrehijo de dos grupos de mujeres afectadas de psicosis endógena o no-endógena
TAT; WISC; WAIS;

SADS; Rorschach; Bender-Gestalt;

Human Figure

Drawings; DSM-I;

DSM-III; Benjamin

Proverbs Test;

World Association Test; MMPI

GAS; Social

Competence Index; Role Functioning

Scale; Shipley-

Hartford intelligence

test; Mother's

Child.Rearinr

Problem Solving

Test (MOPS); Bayley

Scales; McCarthy

Scales; Infant

Temperament

Questionnaire (ITQ);

Toddler

Temperament

Questionnaire

(TTQ): Behavioral

Style Questionnaire

(BSQ); Children's

Self-Concept Test;

Preschool

Interpersonal

Problem Solving

Scale (PIPS); HOME

Inventory; DSM-III

RDC; Griffiths'

Developmental

Scale; Children's

Apperception Test;

CPT; Modified

Rochester

Adaptative

Behavior Inventory

(RABI); Children's

Global Assessment

Scale (CGAS);

DSM-III
- La PDM se relaciona signif. con la SQZ materna;

- La gravedad de la PDM se asocia a la de la psicopatología hallada a los 10 años de edad

- La descendencia de madres esquizofrénicas tiene un funcionamiento global peor.

- Las madres depresivas son menos competentes que las demás, y junto con las SQZ son menos cariñosas y estimuladoras.

Los trastornos infantiles a los 6 años se encuentran asociados a:

- ansiedad en el embarazo

- psicosis de la madre de los 6 meses a los 2 años post-parto

- actitud negativa o ambivalente hacia el embarazo 
Tabla 3. (Continuación)

\begin{tabular}{ll}
\hline \multicolumn{1}{c}{ Estudio } & Diseño del estudio \\
\hline $\begin{array}{l}\text { Sameroff et al. } \\
\text { (1987) }\end{array}$ & Evaluación \\
& longitudinal (0 a 4 \\
& años) del desarrollo \\
& cognitivo, \\
& psicomotor, \\
& emocional y social \\
& de 184 hijos de \\
& madres SQZ, \\
& depresivas, con trs. \\
& de personalidad y \\
& sanas
\end{tabular}

Mednick, Parnas y Schulsinger (1987)

Seguimiento de 207 niños de alto riesgo genético de SQZ y 104 controles

Objetivos/Hipótesis

Evaluar el impacto

en la descendencia

de las VV:

a) diagnóstico materno

b) gravedad de este diagn.

c) estatus social

Valoración del riesgo que implican: 1) antecedentes biológicos de SQZ

2) traumas perinatales 3) inestabilidad del ambiente de crlanza

\begin{tabular}{|c|c|}
\hline Evaluaciones & Resultados \\
\hline $\begin{array}{l}\text { Bayley MDI, PDI y } \\
\text { IBR; RABI; } \\
\text { Development of } \\
\text { Visual Pursuit and } \\
\text { Permanence } \\
\text { Objects Scale; } \\
\text { WPPSI; Vineland } \\
\text { Social Maturity } \\
\text { Scale; Paternal } \\
\text { Attitude Research } \\
\text { Instrument, EPQ; } \\
\text { DSM-II; DSM-III }\end{array}$ & $\begin{array}{l}\text { - La SQZ materna } \\
\text { tiene el menor } \\
\text { impacto de todas } \\
\text { las variables } \\
\text { - Los niños } \\
\text { expuestos a } \\
\text { mayor riesgo } \\
\text { ambiental tienen } \\
\text { peor pronóstico }\end{array}$ \\
\hline $\begin{array}{l}\text { WISC; medidas } \\
\text { psicofisiologicas y } \\
\text { de personalidad; } \\
\text { Adjective } \\
\text { Checklist, } \\
\text { entrevista } \\
\text { psiquiátrica; } \\
\text { informes escolares } \\
\text { de los maestros; } \\
\text { Midwife's Report } \\
\text { on Subject's } \\
\text { Pregnancy and } \\
\text { Delivery y DSM-III }\end{array}$ & $\begin{array}{l}\text { Sugieren que el } \\
\text { SPD es lo que se } \\
\text { transmite de } \\
\text { base por via } \\
\text { genética } \\
\text { - Los sujetos de } \\
\text { riesgo muestran } \\
\text { mayor pasividad } \\
\text { y desatención en } \\
\text { la infancia, y } \\
\text { más tr. del } \\
\text { pensamiento y } \\
\text { dificultades de } \\
\text { vinculación en la } \\
\text { adolescencia }\end{array}$ \\
\hline
\end{tabular}
ambientes familiares

Tompson et al. (1997) hijos de madres

SQZ dados en adopción con 135 sujetos adopatados controles

Examen de los trs. del pensamiento y los patrones comunicativos familiares de 42 chicos y 14 chicas de entre 7 y 14 años, de diferentes razas
Hipótesis: la vulnerabilidad genética interactua con el ambiente adoptivo: los predisponentes incrementan la vulnerabilidad y los ambientes protectores la disminuyen
1) Los sjs. con SQZ o SPD exhibiran mayores trs. del pensamiento $y$ problemas de comunicación
WISC-R; C-GAS; Thought Disorder Index (TDI); Family Consensus
Rorschach Task; K- SADS-E
2) Sus padres también manifiestaran mayores problemas comunicativos
3) Existirán diferencias
- Mayores niveles de tr. del pensamiento $y$ problemas comunicativos en niños con trastornos del espectro
- La misma diferencia entre padres de niños con trastornos del espectro y padres de los controles

RDC; PSE; GAS; MMPI; SANS;

Los niños

vulnerables son

SCID-II; DSM-III; más sensibles a los

Oulu Family Rating ambientes

Scale (OPAS); familiares

Rorschach

disruptivos que los controles entre sjs. con SPD y controles 
Tabla 3. (Continuación)

\begin{tabular}{|c|c|c|c|c|}
\hline Estudio & Diseño del estudio & Objetivos/Hipótesis & Evaluaciones & Resultados \\
\hline $\begin{array}{l}\text { Modestin, Oberson } \\
\text { y Erni (1998) }\end{array}$ & $\begin{array}{l}\text { Retrospectivo. } \\
\text { Analizan el } \\
\text { ambiente y } \\
\text { relaciones } \\
\text { familiares de } 90 \\
\text { pacientes de ambos } \\
\text { sexos con trs. de la } \\
\text { personalidad }\end{array}$ & $\begin{array}{l}\text { Los } \\
\text { acontecimientos } \\
\text { estresantes, las } \\
\text { disputas familiares } \\
\text { y la vinculación } \\
\text { con los padres } \\
\text { incide en el } \\
\text { desarrollo posterior } \\
\text { de patología }\end{array}$ & $\begin{array}{l}\text { SCID-II-PQ; PDE; } \\
\text { The Parental } \\
\text { Bonding } \\
\text { Instrument (PBI) }\end{array}$ & $\begin{array}{l}\text { - El } 23 \% \text { de los } \\
\text { sujetos cursa trs. } \\
\text { de personalidad } \\
\text { del tipo A, pero } \\
\text { sólo } 6 \text { de los } 90 \\
\text { cursan SPD. } \\
\text { - La influencia del } \\
\text { ambiente casero } \\
\text { depende del } \\
\text { sexo }\end{array}$ \\
\hline
\end{tabular}

Abreviaciones: CPT (Continuous Perfomance Test); Cticas. (Características); EPQ (Eysenck Personality Questionnaire); GAS (Global Assessment Scale); IBR (Infant Behavior Record); K-SADS-E (Shedule for Affective Disorders and Scizophrenia for School Age Children-Epidemiological version); MDI (Mental Development Index); PDI (Psychomotor Development Index); PSE (Present State Examination); RABI (Rochester Adaptative Behavior Inventory); RDC (Research Diagnostic Criteria); SADS (Shedule for Affective Ddisorders and Schizophrenia); Sjs. (sujetos); TAT (Thematic Apperception Test); VV. (variables); WAIS (Wechsler Adult Intelligence Scale); WISC (Wechsler Intelligence Scale for Children); WPPSI (Wechsler Preschool and Primary Scale of Intelligence).

Una madre negligente puede acentuar la vulnerabilidad genética de su hijo a los trastornos del espectro esquizofrénico. Aunque con respecto al niño su conducta es una variable ambiental, en el ámbito de los precursores no deja de constituir un comportamiento, así que esta actitud materna puede entenderse como un precursor conductual. La relación del niño de riesgo con las personas con las que establece un vínculo afectivo, constituye igualmente una variable marcadora en tanto que ciertas alteraciones relacionales sensibilizan la predisposición constitucional del niño a expresar psicosis. También las percepciones infantiles de la armonía familiar constituyen un potencial estresor ante el riesgo de psicosis, dado que el impacto del ambiente disruptivo en un niño vulnerable puede conducir a la eclosión de un cuadro esquizofrénico.

Variables de este tipo son las que merecen atención en este punto, pues aunque no son manifestaciones de un riesgo heredado pueden contribuir a que, de existir, se manifieste. En este sentido, los trabajos de Mednick, Parnas y Schulsinger (1987) sobre la muestra danesa permiten establecer diferencias entre los sujetos de riesgo que desarrollan un cuadro esquizofrénico y los que no. Según ellos, todos los sujetos vulnerables exhiben un funcionamiento "esquizotáxico» en la infancia (pasividad, desatención, trastornos formales del pensamiento, vínculos defectuosos) pero los que se descompensan en la adultez parecen haber sufrido más experiencias desafortunadas tempranas tales como insatisfacción e inestabilidad en la relación con sus padres.

Modestin, Oberson y Erni (1998) apoyan el valor etiopatogénico de las relaciones paternas en los trastornos de la personalidad, distinguiendo antecedentes diferentes en función del sexo: si bien la relación con el padre correlaciona positivamente con los trastornos de la personalidad de los chicos, las variables clave en el caso de las chicas son la sobreprotección materna y el presenciar violencia en el hogar.

Según Tompson et al. (1997), las madres de niños con trastornos del espectro esquizofrénico manifiestan mayores niveles de trastorno del pensamiento y dificultades en la comunicación que las madres de niños depresivos y las de niños sanos. Paradojicamente, las madres del grupo control son las que más 
tienden a desviar la atención, pero su distractibilidad es interpretada más como un indicio de relajación en las relaciones interpersonales que como un problema atencional.

La psicopatología hallada a los 6 años en la descendencia de riesgo guarda relación con la ansiedad y la actitud maternas con respecto al embarazo, así como con la condición psicótica de la madre entre los 6 meses y los 2 años después del parto (McNeil y Kaij, 1987). Los niños menos competentes a los 5 años descienden de madres más gravemente trastornadas, menos hábiles resolviendo los problemas de crianza y más inestables en el ambiente educativo que proporcionan a sus hijos (Goodman, 1987). No obstante, en los casos en los que el niño es dado en adopción, el ambiente adoptivo puede protegerle o hacerle más vulnerable. En este sentido, la literatura coincide en señalar que los niños de riesgo (dados en adopción) que cursan cuadros del espectro esquizofrénico han crecido en familias adoptivas seriamente perturbadas (Tienari et al. 1987; 1994).

Aparentemente, la cualidad de precursor de las actitudes parentales es una prueba en favor de la validez del modelo etiológico interactivo herencia-ambiente. Algunos autores encuentran que el desarrollo infantil se ve más perjudicado por una madre neurótico-depresiva que por una madre biológica esquizofrénica (Sameroff, Seifer, Zax y Barocas, 1987; Tienari et al. 1994). Ello podría estar enfatizando la importancia del factor ambiental como precipitante.

Por otra parte, cabe destacar, entre los estudios revisados, los que evidencian la prontitud con que se manifiestan algunos signos prodrómicos, sugiriendo cuán temprano es posible cribar a los sujetos susceptibles de intervención preventiva.

En esta linea, Fish (1987) intentó verificar la hipótesis de la «pandysmaturation» comparando a doce hijos de madre esquizofrénica con doce controles. Hipotetizó que la pandismaduración o déficit neurointegrativo puede ser marcador infantil de un rasgo esquizotípico inherente. La existencia de correlación significativa entre la gravedad del déficit y la esquizofrenia materna permite descartar la posibilidad de que se trate de un precursor inespecífico de psicopatología en general. Además, la gravedad de esta alteración también correlaciona con la psicopatología del niño a los 10 años. Hay que tener en cuenta que la mitad de los niños de riesgo serán diagnosticados de personalidad esquizotípica o paranoide tras manifestar aislamiento, soledad, retracción social y depresión, así como impulsividad, y déficit perceptivos entre los 3 y los 6 años, y cabe señalar que muchos de estos análogos de la sintomatología psicótica negativa cuentan con antecedentes antes de los 2 años de edad.

Otro signo prodrómico con continuidad entre la psicopatología hallada a los 10 años y la evaluación del trastorno mental entre los 15 y los 22 parece ser el trastorno del pensamiento. Fish incluye, en los diagnósticos de la psicopatología preadolescente de estos niños, el registro de sutiles indicadores de errores en el pensamiento lógico y la evaluación de la realidad. Mediante el WISC y el Rorschach, corrobora la capacidad del "Thought Disorder Index» - TDI(Johntson y Holzman, 1979) como predictor del trastorno esquizotípico diagnosticado posteriormente.

A su tiempo, Tompson et al. (1997), mediante el mismo índice, verificaron la asociación entre el pensamiento trastornado y la esquizotipia al encontrar niveles de éste significativamente mayores en 20 sujetos diagnosticados de trastornos del espectro esquizofrénico (10 SQZ y 10 $\mathrm{SPD}$ ), que en 18 controles de las mismas edades (entre 7 y 14 años).

Goodman (1987) siguió el desarrollo social e intelectual de 71 descendientes 
biológicos de madres esquizofrénicas, mostrando que estos expresan menor competencia comunicativa y obtienen menores puntuaciones en "role playing" de expresión de los afectos negativos (hostilidad, enfado) y de utilización de la madre como recurso que el grupo control. Además, mediante un índice de desarrollo (IQMD) compuesto por las puntuaciones de las escalas de Bayley y McCarthy evidencia la diferencia en eficacia intelectual entre la descendencia de riesgo (media 85) y los hijos de madre sana (media 97). El hecho de que Goodman haya estudiado los cinco primeros años de vida de estos niños permite atribuir mayor valor a la capacidad discriminativa de estas variables, que pueden detectar al sujeto vulnerable antes del fin de la etapa preescolar.

\section{Trastorno esquizotípico de la} personalidad versus esquizofrenia

$¿$ Cabe distinguir precursores diferentes para la esquizofrenia y el trastorno esquizotípico? ¿Es posible que los sujetos adultos diagnosticados de trastorno esquizotípico de la personalidad, se comportaran, en su infancia, de forma diferente a los diagnosticados de esquizofrenia? A pesar de la afinidad fenomenológica existente entre dos síndromes pertenecientes al mismo espectro psicopatológico, diferentes trabajos coinciden en corroborar esta distinción.

La muestra danesa permite la extracción de conclusiones al respecto. Para empezar, tanto los sujetos diagnosticados de esquizofrenia en la adultez como sus padres, percibían las relaciones familiares como significativamente menos satisfactorias que lo que reportaban tanto los sujetos diagnosticados de trastorno esquizotípico como sus padres (Burman, Mednick, Machón, Parnas y Schulsinger, 1987). En segundo lugar, aunque la carga genética y sus manifestaciones tempranas en forma de "déficit neurointegrativo" son comparables en ambos grupos de riesgo, los esquizofrénicos (sobre todo los chicos) evidencian una afectividad y control de impulsos significativamente más pobres que los esquizotípicos (Mednick, Parnas y Schulsinger, 1987). Además, los esquizofrénicos tienen menor contacto con sus padres y madres, sufren más frecuentemente las consecuencias de la institucionalización y presentan más traumas perinatales posteriormente asociados a atrofia cerebral.

Las consecuencias del daño cerebral ya son aludidas en análisis previos de esta muestra. John, Mednick y Schulsinger (1982) afirmaron que esquizofrénicos y esquizotípicos aparecen como solitarios, aislados y socialmente ineptos en su infancia. También son niños ansiosos y tensos, pero si algo distingue a los dos grupos es la dificultad de los esquizofrénicos para inhibir su comportamiento disruptivo en la escuela, dificultad hipotéticamente vinculada al daño cerebral perinatal.

Estos mismos autores distinguen diferencias comportamentales en función del sexo. Si bien los futuros esquizotípicos son niños anhedónicos, aislados y distantes según sus maestros, los futuros esquizofrénicos son más disruptivos e indisciplinados. De entre las chicas, las futuras esquizofrénicas exhiben mayor descontrol que las futuras esquizotípicas, en conjunto más reservadas. Estas diferencias son complementadas por hallazgos posteriores, que confirman la mayor agresividad de los futuros esquizofrénicos y añaden, a la reserva manifestada por las futuras esquizotípicas, una sensibilidad y dificultad para encajar la crítica superiores a las de las futuras esquizofrénicas (Olin y Mednick, 1996).

Así, aunque existe evidencia de la relación factorial entre las dimensiones de la esquizofrenia y las de la esquizoti- 
pia (Gruzelier, 1996), las diferencias entre comportamientos precursores de una y otra abarcan la multidimensionalidad demostrada de ambos sindromes (Tyrka et al. 1995; Chen, Hsiao y Kin, 1997; Kravetz, Faust y Edelman, 1998; Wolfradt y Straube, 1998). Además, puesto que parece claro que la estructura factorial de la esquizotipia es la misma en ambos sexos (Olin et al. 1997) es lícito generalizar conclusiones sin distinguir diferencias entre ellos.

Al factorizar el cuestionario mediante el que los maestros evaluaron a esta muestra en 1962, el grupo danés (Olin et al., 1997) descubre que los rasgos esquizotípicos de los 162 niños de alto riesgo evaluados se estructuran en cuatro factores: (1) ansiedad social e introversión, (2) disruptividad e hiperexcitablilidad, (3) pasividad y despreocupación y (4) hipersensibilidad a la crítica. Aunque ni el primer factor ni el tercero permiten distinguir a los futuros esquizotípicos de los futuros esquizofrénicos, éstos se confirman significativamente más hiperexcitables y disruptivos que aquéllos (factor 2), y menos hipersensibles a la crítica (factor 4). El análisis por sexos confirma, básicamente, los resultados del de la muestra conjunta, con una única diferencia: aunque el único factor que discrimina específicamente a los futuros esquizotípicos de los futuros esquizofrénicos sigue siendo el segundo (disruptividad), las diferencias sólo son significativas en el sexo masculino; las pre-esquizotipicas no son menos disruptivas e hiperexcitables que las pre-esquizofrénicas.

Tres cuartas partes de los pre-esquizotípicos se distinguian de sus compañeros escolares por su conducta. Estos rasgos comportamentales distintivos encuentran correlatos adultos en los criterios diagnósticos del trastorno esquizotípico de la personalidad (DSM-III-R, DSM-IV). Su pasividad y seriedad puede que evolucionen en la constricción afectiva de la esquizotipia adulta, la desvinculación social infantil en la falta de amigos íntimos y la hipersensibilidad a la crítica en ansiedad social excesiva. Aunque éste último rasgo (excesiva ansiedad social) se asocia a ideación paranoide en el DSM$I V$, los pre-esquizotípicos no aparentan ser más socialmente ansiosos que el resto de niños, razón por la que los autores hipotetizan que la hipersensibilidad y pasividad de estos niños les conduciria a restringir sus oportunidades de socialización, condicionando así el surgimiento posterior del trasfondo ansioso, que explicaría su carencia adulta de amigos intimos y confidentes.

La existencia de esta analogía entre signos prodrómicos y manifestaciones morbosas ulteriores no sólo indica que este trastorno no se desarrolla de repente en la adultez, sino que además de arraigar en la infancia, puede que se geste entre influencias de distintos factores, tanto genéticos como ambientales, $y$ tan tempranos como para ejercer su efecto antes de la edad escolar.

\section{DISCUSION Y CONCLUSIONES}

La conclusión a la que invita el conjunto de los resultados es que existen cuadros sintomáticos infantiles análogos a los trastornos del espectro esquizofrénico en la edad adulta. Hallazgos recientes apoyan, incluso, la existencia de entidades posiblemente distintas de las ya conocidas. Es el caso del «trastorno por déficit multidimensional», postulado por Kumra et al. (1998) como posible alternativa a la esquizofrenia de inicio infantil.

Estos signos infantiles, precursores conductuales de trastornos posteriores, sugieren por si mismos los posibles factores protectores. La mayoría de autores coinciden en el valor protector de muchas de estas variables, de las que presentamos una muestra en la tabla 4. 
Tabla 4. Factores protectores bioconductuales y ambientales en el desarrollo de los sujetos de riesgo elevado

\begin{tabular}{|c|c|}
\hline Protectores Bio-Conductuales & Protectores Ambientales \\
\hline $\begin{array}{l}\text { - Sistema Nervioso Central correctamente inte- } \\
\text { grado }\end{array}$ & $\begin{array}{l}\text { - Ambiente familiar sano, libre de perturbacio- } \\
\text { nes relacionales y problemas comunicativos }\end{array}$ \\
\hline - Elevada inteligencia & $\begin{array}{l}\text { - Red sólida de apoyo social en la etapa adoles- } \\
\text { cente y preadolescente }\end{array}$ \\
\hline $\begin{array}{l}\text { - Motivación y actitud perseverante } \\
\text { - Extroversión, sociabilidad y comportamientos } \\
\text { prosociales }\end{array}$ & $\begin{array}{l}\text { Estabilidad en la relación con unos padres } \\
\text { capaces de responder a las necesidades cam- } \\
\text { biantes de un sujeto en constante desarrollo }\end{array}$ \\
\hline $\begin{array}{l}\text { - Ausencia de retraimiento social / Presencia de } \\
\text { sentimientos de pertenencia a un grupo }\end{array}$ & $\begin{array}{l}\text { - Alto nivel de funcionamiento general en los } \\
\text { antecesores directos no enfermos }\end{array}$ \\
\hline \multirow[t]{3}{*}{ - Atractivo físico } & - Que la madre NO presente: \\
\hline & $\begin{array}{l}\text { - trastornos afectivos graves } \\
\text { - pobre competencia social } \\
\text { - pocas habilidades estimulativas }\end{array}$ \\
\hline & - Estatus socioeconómico elevado \\
\hline
\end{tabular}

Centrar los estudios revisados en un marco interpretativo adecuado supone revisar también las limitaciones metodológicas a las que están sometidos y a las que hay que someter sus conclusiones. $\mathrm{El}$ tamaño de las muestras, la prevalencia de los trastornos implicados y la sobresimplificación de los modelos teóricos de partida dificultan la generalización de resultados. Por esta razón, se hace aconsejable aumentar dicho tamaño y no ignorar, en los análisis estadísticos, la contribución de importantes factores que aunque perjudican la simplicidad de los modelos por su complejidad, son indispensables para un acercamiento válido y fiable a la realidad que se pretende descifrar. Omitir estas variables ciertamente permitiría simplificar la explicación de los resultados, pero también conllevaría, inevitablemente, un reduccionismo explicativo que invalidaría la generalización de las conclusiones.

El conjunto de los autores hasta aquí citados reúnen, entre sus aportaciones, algunas recomendaciones para la investigación futura. En primer lugar, prescriben una evaluación exhaustiva de todas las variables relativas al sujeto, desde la historia perinatal completa hasta las observaciones de su comportamiento escolar y experiencias sociales. En segundo lugar, proponen completar el barrido de datos mediante el registro completo del funcionamiento neurologico y cognitivo de los padres, su estatus psicopatológico y la observación de sus patrones relacionales en la dinámica familiar. En tercer lugar, aconsejan disponer de múltiples grupos de riesgo, hijos de padres afectados por diferentes trastornos, comparables demográficamente y cuyos diagnósticos sean realizados a ciegas, garantizando la validez de estas comparaciones. Finalmente, opinan que la colaboración entre centros, organizada en base a un protocolo nuclear, que permita evaluar muestras de distintas razas y culturas sin impedir su fusión posterior en una muestra conjunta, no sólo elevaría la categoría del estudio y la ecología de sus resultados sino que permitiría el desarrollo de estimadores de riesgo diferenciales según las variables de importancia en 
cada subpoblación (Tienari et al. 1987; Marcus et al. 1987; Erlenmeyer-Kimling y Cornblatt 1987; Cannon, Mednick y Parnas, 1990; Maziade et al. 1996).

La detección de un riesgo conlleva unas implicaciones preventivas. La prevención, cuánto más temprana mejor. ¿Cuándo es demasiado tarde? Para Barbara Fish, ya lo es entre los 3 y los 6 años, para prevenir el daño cognitivo y social de los niños genéticamente vulnerables a los trastornos del espectro esquizofrénico.

Así, en respuesta a los interrogantes formulados en la introducción, podemos concluir que, efectivamente, los individuos que desarrollan trastornos del espectro esquizofrénico en la adultez, se comportan de forma diferente a los demás niños en su infancia.

El momento más temprano en que es posible detectar estas diferencias se encuentra en la etapa preescolar, más concretamente entre los 2 y los 4 años, aunque ya antes se insinúan anormalidades en el funcionamiento del sensorio, la motricidad y la capacidad para atender, así como temperamento difícil, pasividad, inhibición y falta de espontaneidad. También las madres de estos niños exhiben menor competencia comunicativa en la interacción con ellos, negligencia en el cuidado de sus necesidades y ansiedad durante los 2 primeros años posparto.

Finalmente, ¿cuál es la naturaleza de esas manifestaciones de vulnerabilidad? Su variabilidad oscila entre los déficit del funcionamiento neurointegrativo y los estresores ambientales, pasando por las alteraciones psiquiátricas y del funcionamiento social. Además, se transmiten análogamente a lo largo del desarrollo hasta cobrar la categoría de criterios diagnósticos en la adultez. Lo que entre los 2 y los 4 años se insinúa como alteraciones de la coordinación viso-motriz, se va agravando a medida que se convierte en manifestaciones más graves de trastorno neuroló- gico hasta que es medible, a partir de la adolescencia, mediante el WISC-R y el WAIS. La sintomatología ansioso-depresiva y el comportamiento esquizoide de los 3 años, conducen a los déficit en la competencia social de la infancia media antes de deteriorar definitivamente las relaciones interpersonales y el ajuste social del joven adulto. Por su parte, la susceptibilidad preescolar a recibir un diagnóstico de trastorno del desarrollo se consolida en sintomatología infantil, positiva y negativa, hasta reunir los criterios de un trastorno de conducta grave - aunque no psicótico- en la adolescencia. Finalmente, las alteraciones perceptivas y atencionales inicialmente premórbidas se presentan asociadas a los sintomas esquizotípicos y los trastornos del espectro esquizofrénico que permitieron preveer.

\section{REFERENCIAS BIBLIOGRÁFICAS}

Altman, H., Collins, M. y Mundy, P. (1997). Subclinical hallucinations and delusions in nonpsychotic adolescents. Journal of Child Psychology and Psychiatry, 38, 413 420.

Apter, A., Bleich, A. y Tyano, S. (1988). Affective and psychotic psychopathology in hospitalized adolescents. Journal of the American Academy of Child and Adolescent Psychiatry, 27, 116-120.

Bleuler, E. (1950). Dementia praecox or the group of schizophrenias. (J. Zinken, trans.) New York: International Universities Press. (Publicación original, 1911.)

Burman, B., Mednick, S.A., Machón, R.A., Parnas, J. y Schulsinger, F. (1987). Children at igh risk for schizophrenia: parent and offspring perceptions of family relationships. Journal of Abnormal Psychology, 96 , 364-366.

Cannon, T.D., Mednick, S.A. y Parnas, J. (1990). Antecedents of predominantly negative- and predominantly positivesymptom schizophrenia in a high-risk population. Archives of General Psychiatry, 47, 622-632. 
Caplan, R. (1994). Though disorder in childhood. Journal of American Academy of Child and Adolescent Psychiatry, 33, 605615.

Cassady, S.L., Adami, H., Moran, M., Kunkel, R. y Thaker, G.K. (1998). Spontaneus dyskinesia in subjects with schizophrenia spectrum personality. American Journal of Psychiatry, 155, 70-75.

Chapman, J. (1966). The early symptoms of schizophrenia. British Journal of Psychiatry, 112, 225-251.

Chapman, L.J., Chapman, J.L., Kwapil, T.R., Eckblad, M. y Zinfer, M.C. (1994). Putatively psychosis prone subjects ten years later. Journal of Abnormal Psychology, 103, 171-183.

Chen, W.J., Hsiao, Ch.K. y Lin, Ch.C.H. (1997). Schizotypy in Comunity Samples: The three-factor structure and correlation with sustained attention. Journal of Abnormal Psychology, 106, 649-654.

Chen, W.j., Liu, S.K., Chang, Ch.J., Lien, Y.J., Chang, Y.H. y Hwu, H.G. (1998). Sustained attention deficit and schizotypal personality features in nonpsychotic relatives of schizophrenic patients. American Journal of Psychiatry, 155, 1214-1220.

Eckblad, M. y Chapman, J.L. (1983). Magical ideation as an indicator of schizotypy. Journal of Consulting and Clinical Psychology, 51, 215-225.

Erlenmeyer-Kimling, L. y Cornblatt, B. (1987). The New York high-risk project: a followup report. Schizophrenia Bulletin, 13, 451-461.

Erlenmeyer-Kimling, L., Cornblatt, B., Rock, D., Roberts, S., Bell, M. y West, A. (1993). The New York high-risk project: anhedonia, attentional deviance, and psychopathology. Schizophrenia Bulletin, 19, 141-153.

Erlenmeyer-Kimling, L., Squires-Wheeler, E., Adamo, U.H., Basset, A.S., Cornblatt, B., Kestenbaum, C.J., Rock, D., Roberts, S.A. y Gottesman, I.I. (1995). The New York highrisk project. Archives of General Psychiatry, 52, 457-466.

Famularo, R., Kinscheff, R. y Fenton, T. (1992). Psychiatric diagnoses of maltreated children: preliminary findings. Journal of the American Academy of Child and Adolescent Psychiatry, 31, 863-867.
Fish, B. (1987). Infant predictors of the longitudinal course of schizophrenic development. Schizophrenia Bulletin, 13, 395-409.

Gooding, D.C. y Iacono, W.G. (1995); Schizophrenia through the Lens of Developmental Psychopathology Perspective. En D. Ciccetti y D.J. Cohen (Eds.), Developmental Psychopathology, Vol. 2. New York: John Wiley y Sons Inc.

Goodman, S.H. (1987). Emory university project on children of disturbed parents. Schizophrenia Bulletin, 13, 411-423.

Gruzelier, J.H. (1996). The factorial structure of schizotypy: part I. Affinities with syndromes of schizophrenia. Schizophrenia Bulletin, 22, 611-620.

John, R.S., Mednick, S.A. y Schulsinger, F. (1982). Teacher reports as a predictor of schizophrenia and borderline schizophrenia: A Bayesian Decision Analysis. Journal of Abnormal Psychology, 91, 399-413.

Johnston, M.H. y Holzman, P.S. (1979). Assessing Schizophrenic Thinking. San Francisco: Jossey-Bass, Inc.

Kravetz, S., Faust, M. y Edelman, A. (1998). Dimensions of schizotypy and lexical decision in the two hemispheres. Personality and Individual Differences, 25, 857-871.

Kumra, S., Jacobsen L.K., Lenane, M., Zahn, T.P., Wiggs, E., Alaghband-Rad, J., Castellanos, F.X., Frazier, J.A., McKenna, K., Gordon, Ch.T., Smith, A., Hamburger, S. y Rapoport, J.L. (1998). Multidimensionally impaired disorder: Is it a variant of very early-onset schizophrenia?. Journal of American Academy of Child and Adolescent Psychiatry, 37, 91-99.

Kwapil, T.R. (1998). Social anhedonia as a predictor of the development of schizophrenia-spectrum disorders. Journal of Abnormal Psychology, 107, 558-565.

Lencz, T., Raine, A., Scerbo, A., Redmon, M., Brodish, S., Holt, L. y Bird, L. (1993). Impaired eye tracking in undergraduates with schizotypal personality disorder. American Journal of Psychiatry, 150, 152-153.

Livingston, R., Lawson, L. y Jones, J.G. (1993). Predictors of self-reported psychopathology in children abused repeatedly by parent. Journal of the American Academy of Child and Adolescent Psychiatry, 26, 948-953. 
Maier, W., Lichtermann, D., Mingues, J. y Heun, R. (1994). Personality disorders among relatives of chizophrenic patients. Schizophrenia Bulletin, 20, 481-493.

Marcus, J., L. Hans, S.L., Nagler, S., Auerbach, J.G., Mirsky, A.F. y Aubrey, A. (1987). Review of the NIMH Israeli Kibbutz-City Study and the Jerusalem Infant Development Study. Schizophrenia Bulletin, 13, 425-437.

Martínez, P.C., Lemos, S. y Bobes, J. (1999). Indicadores cognitivos en los trastornos del espectro esquizofrénico: en busca de nuevos marcadores. En J. Saiz (ed.), La esquizofrenia: enfermedad del cerebro $y$ reto social (pp. 83-106). Barcelona: Masson, Colección Psiquiatría Médica.

Maziade, M., Bouchard, S., Gingras, N., Charron, L., Cardinal, A., Roy, M.A., Gauthier, B., Tremblay, G., Côté, S., Fournier, C., Boutin, P., Hamel, M., Mérette, Ch. y Martínez, M. (1996). Long-term stability of diagnosis and symptom dimensions in a systematic sample of patients with onset of schizophrenia in childhood and early adolescence II: positive/negative distinction and childhood predictors of adult outcome. British Journal of Psychiatry, 169, 371-378.

McNeil, T. F. y Kaij, L. (1987). Swedish HighRisk Study: sample characteristics at age 6. Schizophrenia Bulletin, 13, 373-381.

Mednick, S.A., Parnas, J. y Schulsinger, F. (1987). The Copenhagen high-risk project, 1962-86. Schizophrenia Bulletin, 13, 485495.

Meehl, P.E. (1962). Schizotaxia, schizotypy, schizophrenia. American Psychologist, 17, 827-838.

Meehl, P.E. (1989). Schizotaxia Revised. Archives of General Psychiatry, 46, 935-944.

Meehl, P.E. (1990). Toward an integrated theory of schizotaxia, schizotypy and schizophrenia. Journal of Personality Disorders, 4, 1-99.

Modestin, J., Oberson, B., Erni, T. (1998). Possible antecedents of DSM-III-R personality disorders. Acta Psychlatrica Scandinavica, 97, 260-266.

Moritz, S.H., Mass, R. y Junk, U. (1998). Further evidence of reduced negative priming in positive schizotypy. Personality and Individual Differences, 24, 521-530.
Obiols, J.E., Serrano, F., Barrantes, N., García, M., Gras, E., Bosch, S., Caparrós, B. y Carandell, F. (1997). Frontal dysfunction and psychosis proneness in CPT-linked vulnerable adolescents. Personality and Individual Differences, 23, 677-683.

Obiols, J.E., Barrantes, N., Serrano, F., Caparrós, B. y Subirà, S.. Soft neurological signs in CPT-linked vulnerable adolescents: markers of liability for schizophrenia spectrum disorders? Psychiatry Research (En prensa).

O'Driscoll, G.A., Lenzenweger, M.F., Holzman, P.S. (1998). Antisaccades and smooth pursuit eye tracking and schizotypy. Archives of General Psychiatry, 55, 837-843.

Olin, S.S. y Mednick, S.A. (1996). Risk factors of psychosis: identifying vulnerable populations premorbidly. Schizophrenia Bulletin, 22, 223-240.

Olin, S.S., Raine, A., Cannon, T.D., Parnas, J., Schulsinger, F. y Mednick, S.A. (1997). Childhood behavior precursors of schizotypal personality disorder. Schizophrenia Bulletin, 23, 93-103.

Parnas, J., Cannon, T.D., Jacobsen, B., Schulsinger, H., Schulsinger, F. y Mednick, S.A. (1993). Lifetime DSM-III-R diagnostic outcomes in the offspring of schizophrenic mothers. Results from the Copenhagen high-risk study. Archives of General Psychiatry, 50, 707-714.

Rado, S. (1953). Dynamics and classification of disordered behavior. American Journal of Psychiatry, 110, 406-416.

Ritsner, M., Karas, S. y Ginath, Y. (1993). Relatedness of schizotypal personality to schizophrenic disorders: multifactorial threshold model. Journal of Psychiatry Research, 27, 27-38.

Rosenbaum, J. (1988). Children at risk for schizophrenia: converging lines of evidence. Schizophrenla Bulletin, 14, 613-631.

Rothstein, A. (1981). Hallucinatory phenomena in childhood: a critique of the literature. Journal of American Academy of Child Psychiatry, 20, 623-635.

Russell, A.T. (1992), Schizophrenia. In S.R. Hooper y G.W. Hynd (eds.), Assessment and diagnosis of child and adolescent psychiatric disorders: current issues and procedures. Hillsdale, NJ: Erlbaum. 
Sameroff, A., Seifer, R., Zax, M. y Barocas, R. (1987). Early indicators of developmental risk: rochester longitudinal study. Schizophrenia Bulletin, 13, 383-395.

Schulsinger, H. (1976). A ten-year follow-up of children of schizophrenic mothers. Clinical assessment. Acta Psychiatrica Scandinavica, 53, 371-386.

Siever, L.J., Keefe, R. y Bernstein, D.P. (1991). Dr. Siever and colleagues reply. American Journal of Psychiatry, 148, 1097.

Siever, L.J., Keefe, R., Bernstein, D.P., Coccaro, E.F., Klar, H.M., Zemishlany, Z., Peterson, A.E., Davidson, M., Mahon, T., Horvath, T. y Mohs, R. (1990). Eye tracking impairment in clinically identified patients with schizotypal personality disorder. American Journal of Psychiatry, 147, 740-745.

Strauss, J.S. (1969). Hallucinations and delusions as points on continua function. Archives of General Psychiatry, 21, 581-586.

Thaker, G., Moran, M., Cassady, S., Adami, H. y Tamminga, C. (1991). Normal smooth pursuit eye movements in volunteer subjects meeting schizotypal personality disorder criteria (letter). American Journal of Psychiatry, 148, 1096-1097.

Tienari, P., Sorri, A., Lahti, L., Naarala, M., Wahlberg, K.-E., Moring, J., Pohjola, J. y Wynne, L.C. (1987). Genetic and psychosocial factors in schizophrenia: the finnish adoptive family study. Schizophrenia Bulletin, 13, 477-484.

Tienari, P., Wynne, L.C., Moring, J., Lahti, I., Naarala, M., Sorri, A., Wahlberg, K.-E., Saarento, O., Seitamaa, M., Kaleva, M. y Läksy, K. (1994). The finnish adoptive family study of schixophrenia. Implications for family research. British Journal of Psychiatry, 164, 20-26.

Tompson, M.C., Asarnow, J.R., Hamilton, E.B., Newell, LR.E. y Goldstein, M. (1997). Children with schizophrenia-spectrum disorders: thought disorder and communication problems in a family interactional context. Journal of Child Psychology and Psychiatry, 38, 421-429.
Torgersen, S. (1994). Personality deviations within the schizophrenia spectrum. Acta Psychiatrica Scandinavica, 90, 40-44.

Torgersen, S., Onstad, S., Skre, I., Edvarsen, J. y Kringlen, E. (1993). "True" schizotypal personality disorder: a study of co-twins and relatives of schizophrenic probands. American Journal of Psychiatry, 150, 16611667.

Tyrka, A.R., Cannon, T.D., Haslam, N., Mednick, S.A., Schulsinger, F., Schulsinger, H. y Parnas, J. (1995). The latent structure of schizotypy: I. Premorbid indicators of a taxon of individuals at risk for schizophrenia-spectrum disorders. Journal of Abnormal Psychology, 104, 173-183.

Walker, E. y Lewine, R.J. (1990). Prediction of adult-onset schizophrenia from childhood home movies of the patients. American Journal of Psychiatry, 147, 1052-1056.

Watt, N.F. y Lubensky, A.W. (197.6). Childhood roots of schizophrenia. Journal of Consulting and Clinical Psychology, 44, 363-375.

Watt, N.F., Grubb, T.W. y Erlenmeyer-Kimling, L. (1982). Social, emotional, and intellectual behavior at school among children at high risk for schizophrenia. Journal of Consulting and Clinical Psychology, 50, 171-181.

Williams, L. y Beech, A. (1997). Investigations of cognitive inhibitory processes in schizotypy and schizophrenia. En G. Claridge (ed.), Schizotypy. Implications for Illness and Health. New York: Oxford University Press.

Wolff, S. (1991). 'Schizoid' personality in childhood and adult life II: Adjustment and the continuity with schizotypal personality disorder. British Journal of Psychiatry, 159, 620-629.

Wolfradt, U. y Straube, E.R. (1998). Factor structure of schizotypal traits among adolescents. Personality and Individual Differences, 24, 201-206.

Yeung, A.S., Lyons, M.J., Waternaux, C.M., Faraone, S.V. y Tsuang, M.T. (1993). A family study of self-reported personality traits and DSM-III-R personality disorders. Psychiatry Research, 48, 243-255. 